\title{
Pontryagin Maximum Principle for Semilinear and Quasilinear Parabolic Equations with Pointwise State Constraints
}

\author{
Bei $\mathrm{Hu}^{1}$ and Jiongmin Yong ${ }^{2}$
}

\begin{abstract}
This paper studies the first order necessary conditions for the optimal controls of semilinear and quasilinear parabolic partial differential equations with pointwise state constraints. Pontryagin type maximum principle is obtained.
\end{abstract}

Keywords. parabolic equations, pointwise state constraints, optimal control, Pontryagin maximum principle, Ekeland variational principle.

AMS(MOS) subject classifications. $49 \mathrm{~K} 20,35 \mathrm{~K} 10,35 \mathrm{~K} 20$.

\section{$\S 1$. Introduction.}

In this paper, we are concerned with the following parabolic equation:

$$
\left\{\begin{array}{l}
y_{t}-\sum_{i, j=1}^{n}\left(a_{i j}(x, t) y_{x_{i}}\right)_{x_{j}}=f(x, t, y, u(t, x)), \quad \text { in } \Omega_{T}, \\
\left.y\right|_{\partial \Omega}=0, \\
\left.y\right|_{t=0}=y_{0}(x), \quad x \in \Omega,
\end{array}\right.
$$

where $a_{i j}$ and $f$ are some given functions, $\Omega_{T}=\Omega \times(0, T)$ with $\Omega \subset \mathbb{R}^{n}$ being a bounded domain and $T>0$ being a given time duration. The function $u(x, t)$ is called the control, which takes value in some separable metric space $U$. The solution $y(x, t)$ to (1.1) (for given $y_{0}(x)$ and $\left.u(x, t)\right)$ is called the state of the system and $y_{0}(x)$ is referred to as the initial state. We set $\mathcal{U}=\left\{u: \Omega_{T} \rightarrow U \mid u\right.$ is measurable $\}$. Under proper conditions (see $\S 2$ ), we have that for any $y_{0} \in C_{0}^{\alpha}(\bar{\Omega})$ and $u \in \mathcal{U},(1.1)$ admits a unique solution $y(x, t)$ which is in

1 Department of Mathematics, University of Notre Dame, Notre Dame, IN 46556. This author was partially supported by US NSF Grant DMS 90-24986 and DMS 92-24935.

2 IMA, University of Minnesota, Minneapolis, MN 55455; on leave from Department of Mathematics, Fudan University, Shanghai 200433, China. This author was partially supported by NSF of China and Fok Ying Tung Education Foundation. 
$C\left(\bar{\Omega}_{T}\right)$ (actually, it is even better, see $\left.\S 2\right)$. Then, we may talk about the state constraint of form:

$$
G(y) \in Q
$$

for some continuously Fréchet differentiable map $G: C_{0}\left(\bar{\Omega}_{T}\right) \rightarrow Z$, where $C_{0}\left(\bar{\Omega}_{T}\right)=\{\eta \in$ $\left.C\left(\bar{\Omega}_{T}\right)|\eta|_{\partial \Omega \times[0, T]}=0\right\}, Z$ is some Banach space and $Q \subset Z$. Let us take a look of two important examples of the above type constraints. First, let $Z=C_{0}\left(\bar{\Omega}_{T}\right)$,

$$
Q=\left\{\eta \in Z \mid \eta(x, t) \leq 0,(x, t) \in \bar{\Omega}_{T}\right\}
$$

and $G(y)=g(x, t, y(x, t))$ for some function $g: \bar{\Omega}_{T} \times \mathbb{R} \rightarrow \mathbb{R}$. Then, (1.2) reads

$$
g(x, t, y(x, t)) \leq 0, \quad(x, t) \in \bar{\Omega}_{T}
$$

Our second example is as follows: We again let $Z=C_{0}\left(\bar{\Omega}_{T}\right)$, and define

$$
Q=\left\{\eta \in Z \mid \eta\left(x_{i}, t_{i}\right)=b_{i}, \quad 1 \leq i \leq m\right\}
$$

for some given (different) points $\left(x_{i}, t_{i}\right) \in \Omega_{T} \bigcup(\Omega \times\{T\})$ and numbers $b_{i}$, and $G=I$, the identity operator on $Z$. Then, (1.2) reads

$$
y\left(x_{i}, t_{i}\right)=b_{i}, \quad 1 \leq i \leq m .
$$

For elliptic equations, similar constraints like (1.4) and (1.6) were considered in [4, 5, 19]. The above two examples all require pointwise behavior of the state $y(x, t)$. There are many other examples covered by (1.2) (see $\S 5)$. It is seen that our state constraint (1.2) is very general.

Now, we introduce the following functional:

$$
J(u)=\int_{\Omega_{T}} f^{0}(x, t, y(x, t), u(x, t)) d x d t
$$

for some function $f^{0}$, where $y(x, t)$ is the solution of $(1.1)$ corresponding to $u$. This is called the cost functional. Next, we set

$$
\mathcal{U}_{a d} \equiv\{u \in \mathcal{U} \mid \text { the corresponding } y \text { satisfies (1.2) }\}
$$


Any element $u \in \mathcal{U}_{a d}$ is called an admissible control. In what follows, we assume that $\mathcal{U}_{a d} \neq \emptyset$. Then, we may state our optimal control problem as follows:

Problem C. Find $\bar{u} \in \mathcal{U}_{a d}$, such that

$$
J(\bar{u})=\inf _{\mathcal{U}_{a d}} J(u)
$$

Whenever such a $\bar{u} \in \mathcal{U}_{a d}$ exists, we call it an optimal control; the corresponding state $\bar{y}$ is called an optimal state and $(\bar{y}, \bar{u})$ is called an optimal pair.

Our goal is to obtain a set of first order necessary conditions for the optimal pairs. This set of conditions is called the Pontryagin maximum principle.

In recent papers $[3,4,5,20]$, the Pontryagin maximum principle was derived for semilinear and quasilinear elliptic partial differential equations with pointwise state constraints (see [2] also). For parabolic equations, abstract evolution equation setting was used a little earlier to obtain similar results $([9,10,14,15,18])$. We note that by using the abstract framework for parabolic equations, people treat the time variable $t$ and the spatial variable $x$ unequally, in the sense that the variable $x$ is "averaged" and actually does not appear explicitly in the whole process. Consequently, some pointwise information of the state $y(x, t)$, like Hölder continuity, values at some particular points $\left(x_{0}, t_{0}\right) \in \Omega_{T}$, are lost. In particular, the problem with the state constraint (1.6) can not be covered by abstract framework. In this paper, we use the idea of $[5]$ (see $[13,20]$ also) to discuss the optimal control problem for parabolic equations without using the abstract evolution equations. By this approach, we retain some pointwise behavior of the state $y(x, t)$. Consequently, we can treat general (pointwise) state constraint (1.2), which contains (1.6) as a special case.

Due to the fact that $U$ is just a separable metric space, only the spike perturbation of the control is allowed when we derive the necessary conditions. On the other hand, the pointwise state constraint is presented. These two together cause the main difficulty in our procedure. The key which overcomes this main difficulty is to find the "Taylor expansion" of the state with respect to the spike variation of the control, in a strong enough topology that is sufficient for us to treat the pointwise constraint. We achieve this by improving a technical lemma found in [5] and using proper estimates for parabolic equations. Once 
this is obtained, we then use the usual procedure of applying Ekeland variational principle to derive the desired conclusion.

We refer the readers to $[17,19]$ for some classical relevant results.

The rest of the paper is organized as follows. In Section 2, we give some preliminary result and state the main result. Section 3 is devoted to proving some technical lemmas. The proof of Pontryagin maximum principle is carried out in Section 4. Some applications are given in Section 5. The result corresponding to quasilinear parabolic equations is briefly discussed in Section 6.

\section{Preliminary and the Main Result.}

Let us first give some assumptions and preliminary results. We let $\Omega \subset \mathbb{R}^{n}$ be a bounded domain with $\partial \Omega \in C^{2}, \Omega_{T}=\Omega \times(0, T), \partial_{p} \Omega_{T}=(\bar{\Omega} \times\{0\}) \bigcup(\partial \Omega \times[0, T])$ be the parabolic boundary of $\Omega_{T}, U$ be a separable metric space. We use $|\cdot|$ or $\|\cdot\|$ (some times with a subscript) as the norm in various spaces, which can be identified from the context. For any measurable set $S \subset \mathbb{R}^{n}$, we use $|S|$ to denote the Lebesgue measure of the set $S$. In what follows, we will denote by $C_{0}\left(\bar{\Omega}_{T}\right) \subset C\left(\bar{\Omega}_{T}\right)$ the set of all continuous function on $\bar{\Omega}_{T}$ which vanish on $\partial \Omega \times[0, T]$, by $C^{\beta, \beta / 2}\left(\bar{\Omega}_{T}\right)$ the set of all continuous functions on $\bar{\Omega}_{T}$ which are Hölder continuous in $(x, t)$ with the exponent $\beta$ in $x$ and $\beta / 2$ in $t(\beta \in(0,1))$ and by $C_{0}(\bar{\Omega})$ the set of all continuous functions on $\bar{\Omega}$ which vanish on $\partial \Omega$.

The following assumptions will be assumed throughout of the paper.

(A1) The function $a_{i j}: \Omega_{T} \rightarrow \mathbb{R}$ is measurable, $a_{i j}=a_{j i}$ and there exist constants $\Lambda>\lambda>0$, such that

$$
\lambda|\xi|^{2} \leq a_{i j}(x, t) \xi_{i} \xi_{j} \leq \Lambda|\xi|^{2}, \quad \text { for a.e. }(x, t) \in \bar{\Omega}_{T}, \quad \xi \in \mathbb{R}^{n}
$$

(A2) The function $f: \bar{\Omega} \times[0, T] \times \mathbb{R} \times U \rightarrow \mathbb{R}$ has the following properties: $f(\cdot, \cdot, y, u)$ is measurable on $\Omega \times[0, T], f(x, t, \cdot, u)$ is in $C^{1}(\mathbb{R})$ with $f(x, t, \cdot, \cdot)$ and $f_{y}(x, t, \cdot, \cdot)$ being continuous on $\mathbb{R} \times U$. There exists a constant $C>0$, such that

$$
f(x, t, y, u) y \leq C\left(|y|^{2}+1\right), \quad \forall(x, t, y, u) \in \bar{\Omega} \times[0, T] \times \mathbb{R} \times U
$$


Moreover, for any $R>0$, there exists an $M_{R}>0$, such that

$$
|f(x, t, y, u)|+\left|f_{y}(x, t, y, u)\right| \leq M_{R}, \quad \forall(x, t, u) \in \bar{\Omega} \times[0, T] \times U,|y| \leq R .
$$

The same conditions, except (2.2), hold for the function $f^{0}: \bar{\Omega} \times[0, T] \times \mathbb{R} \times U \rightarrow \mathbb{R}$.

(A3) $Z$ is a Banach space with the dual $Z^{*}$ being strictly convex. $Q \subset Z$ is convex and closed, and is of finite codimension in $Z$ (see below or [15, Definition 2.2]). The map $G: C_{0}\left(\bar{\Omega}_{T}\right) \rightarrow Z$ is continuously Fréchet differentiable.

Let us make some remarks on (A3). First, a set $Q \subset Z$ is said to be finite codimensional in $Z$ if for some $z_{0} \in Q$, the space $Z_{0}$ spanned by $Q-z_{0} \equiv\left\{z-z_{0} \mid z \in Q\right\}$ is a finite codimensional subspace of $Z$ and the convex hull $\overline{\operatorname{co}}\left(Q-z_{0}\right)$ of $Q-z_{0}$ has a nonempty relative interior in $Z_{0}$. It is not hard to see that the set $Q$ defined by (1.3) has a nonempty interior in $Z$ and hence is of codimension 0 in $Z$; the set $Q$ defined by (1.5) is of codimension $m$ in $Z$.

Next, we consider the case $Z=C_{0}\left(\bar{\Omega}_{T}\right)$. Then, by Hahn-Banach Theorem, for any $\mu \in Z^{*} \equiv C_{0}\left(\bar{\Omega}_{T}\right)^{*}$, there exists a $\tilde{\mu} \in C\left(\bar{\Omega}_{T}\right)^{*}=\mathcal{M}\left(\bar{\Omega}_{T}\right)$ (the set of all Radon measures on $\left.\bar{\Omega}_{T}\right)$, such that $\mu=\left.\tilde{\mu}\right|_{C_{0}\left(\bar{\Omega}_{T}\right)}$ and

$$
\langle\tilde{\mu}, \eta\rangle=\int_{\bar{\Omega}_{T}} \eta d \tilde{\mu}, \quad \forall \eta \in C\left(\bar{\Omega}_{T}\right)
$$

Then, for any $\eta \in C_{0}\left(\bar{\Omega}_{T}\right)$ (note $\left.\left.\eta\right|_{\partial \Omega}=0\right)$

$$
\langle\mu, \eta\rangle=\int_{\bar{\Omega}_{T}} \eta d \mu=\int_{\Omega_{T} \bigcup(\Omega \times\{0, T\})} \eta d \mu .
$$

In what follows, we let $\mathcal{M}_{0}\left(\bar{\Omega}_{T}\right)$ be the set of all Radon measures on $\bar{\Omega}_{T}$ with the support contained in $\Omega_{T} \bigcup(\Omega \times\{0, T\})$. Clearly, $\mathcal{M}_{0}\left(\bar{\Omega}_{T}\right)=C_{0}\left(\bar{\Omega}_{T}\right)^{*}$ with the identification being (2.5). It is known that if we use the usual norm in $C_{0}\left(\bar{\Omega}_{T}\right)$, the dual $C_{0}\left(\bar{\Omega}_{T}\right)^{*}$ of it is not strictly convex. However, since the space $C_{0}\left(\bar{\Omega}_{T}\right)$ is a separable Banach space, by [7, p.167], there exists a norm, denoted by $|\cdot|_{0}$, which is equivalent to the norm $\|\cdot\|_{C_{0}\left(\bar{\Omega}_{T}\right)}$, such that the dual of $\left(C_{0}\left(\bar{\Omega}_{T}\right),|\cdot|_{0}\right)$ is strictly convex. It is clear that any element $\mu \in\left(C_{0}\left(\bar{\Omega}_{T}\right),|\cdot|_{0}\right)^{*}$ can still be identified with an element of $\mathcal{M}_{0}\left(\bar{\Omega}_{T}\right)$, such that $(2.5)$ holds. This will be useful when we discuss the case with $Z=C\left(\bar{\Omega}_{T}\right)$ (see $\left.\S 5\right)$. 
Next, we define

$$
d_{Q}(\eta)=\inf _{\eta \in Q}|z-\eta|, \quad \forall \eta \in Z
$$

Then, $d_{Q}: Z \rightarrow \mathbb{R}$ is convex and Lipschitz continuous (with the Lipschitz constant being 1). From [6], we know that the Clarke's generalized gradient, denoted by $\partial d_{Q}$, which coincides with the subdifferential in the sense of the convex analysis in this case [6, Proposition 2.2.7], is convex and weak*-compact. Therefore, given $\xi \in \partial d_{Q}(\eta)$, we have that

$$
\langle\xi, z-\eta\rangle+d_{Q}(\eta) \leq d_{Q}(z), \quad \forall z \in Z
$$

This implies that $|\langle\xi, z-\eta\rangle| \leq|z-\eta|$, for all $z \in Z$, since $d_{Q}(\cdot)$ is Lipschitz continuous with Lipschitz constant 1 . Thus, $\|\xi\|_{Z^{*}} \leq 1$. The identity $\|\xi\|_{Z^{*}}=1$ being true whenever $\eta \notin Q ;$ see $\left[15\right.$, Lemma 3.4]. Since $Z^{*}$ is strictly convex, $\partial d_{Q}(\eta)$ is a singleton for every $\eta \notin Q\left[15\right.$, Corollary 3.5]. Furthermore, $d_{Q}: Z \rightarrow \mathbb{R}$ is Gâteaux differentiable at every point $\eta \notin Q$ and $\left\{\nabla d_{Q}(\eta)\right\}=\partial d_{Q}(\eta)\left[6\right.$, Proposition 2.2.4], where $\nabla d_{Q}(\eta)$ is the Gâteaux derivative of $d_{Q}(\eta)$ at $\eta$. Hence

$$
\left\|\nabla d_{Q}(\eta)\right\|_{Z^{*}}=1, \quad \forall \eta \notin Q
$$

The following result is basic.

Proposition 2.1. Let $(A 1)-(A 2)$ hold. Then, for any $u \in \mathcal{U}$ and $y_{0} \in C^{\alpha}(\bar{\Omega}) \bigcap C_{0}(\bar{\Omega})$ $(0<\alpha<1)$, there exists a $\beta \in(0,1)$, such that (1.1) has a unique solution $y \equiv y(\cdot, \cdot ; u) \in$ $C^{\beta, \beta / 2}\left(\bar{\Omega}_{T}\right) \bigcap L^{2}\left(0, T ; H_{0}^{1}(\Omega)\right)$. Furthermore, there exists a constant $C>0$, independent of $u \in \mathcal{U}$, such that

$$
\|y(\cdot, \cdot ; u)\|_{C^{\beta, \beta / 2}\left(\bar{\Omega}_{T}\right)} \leq C, \quad \forall u \in \mathcal{U}
$$

Sketch of the Proof. Uniqueness follows immediately from the energy estimates. For the existence, it suffices to establish the a-priori estimates for the solution. The assumption (2.2) immediately gives us the $L^{\infty}\left(\Omega_{T}\right)$ estimates. Then the standard energy inequality gives $L^{2}\left(0, T ; H^{1}(\Omega)\right)$ estimates, and the existence of the solution follows. The estimates (2.9) is standard and can be found in [12, Chapter III, section 10]. 
In what follows, any pair $(y, u) \in\left(C^{\beta, \beta / 2}\left(\bar{\Omega}_{T}\right) \bigcap C_{0}\left(\bar{\Omega}_{T}\right)\right) \times \mathcal{U}$ satisfying $(1.1)$ is called a feasible pair and we refer to the corresponding $y$ and $u$ as feasible state and control, respectively. Clearly, under $(\mathrm{A} 1)-(\mathrm{A} 2), \mathcal{U}$ coincides with the set of all feasible controls and for each feasible control $u \in \mathcal{U}$ there corresponds a unique feasible state. Also, we see that the cost functional $J(u)$ is well-defined for each $u \in \mathcal{U}$ and the state constraint (1.2) clearly makes sense.

Now, we assume that the set $\mathcal{U}_{a d}$ defined in (1.8) is nonempty and there exists an optimal pair $(\bar{y}, \bar{u})$ to Problem C. Our main result then can be stated as follows:

Theorem 2.2. (Maximum Principle) Let (A1)-(A3) hold and let the following compatibility condition, for the set $Q$, the map $G$ and the initial state $y_{0}$, holds:

$$
\begin{aligned}
& \operatorname{supp} G^{\prime}(\eta)^{*} \partial d_{Q}(G(\eta)) \subset \Omega_{T} \bigcup(\Omega \times\{T\}) \\
& \forall \eta \in C_{0}\left(\bar{\Omega}_{T}\right) \text { with } G(\eta) \in Q,\left.\quad \eta\right|_{t=0}=y_{0}(x) .
\end{aligned}
$$

Let $(\bar{y}, \bar{u})$ be an optimal pair of Problem $C$. Then, there exists a constant $\psi^{0} \leq 0$, a function $\psi \in L^{q}\left(0, T ; W_{0}^{1, q}(\Omega)\right)\left(1<q<\frac{n+2}{n+1}\right)$, and a $\varphi \in \partial d_{Q}(G(\bar{y})) \subset Z^{*}$, such that

$$
\left\{\begin{array}{l}
\psi_{t}+\sum_{i, j=1}^{n}\left(a_{i j}(x, t) \psi_{x_{j}}\right)_{x_{i}}=-f_{y}(x, t, \bar{y}(x, t), \bar{u}(x, t)) \psi \\
\quad-\psi^{0} f_{y}^{0}(x, t, \bar{y}(x, t), \bar{u}(x, t))+\left.\left(G^{\prime}(\bar{y})^{*} \varphi\right)\right|_{\Omega_{T}}, \quad \text { in } \Omega_{T} \\
\left.\psi\right|_{\partial \Omega}=0 \\
\left.\psi\right|_{t=T}=\left.\left(G^{\prime}(\bar{y})^{*} \varphi\right)\right|_{\Omega \times\{T\}},
\end{array}\right.
$$

$$
\begin{gathered}
\langle z-G(\bar{y}), \varphi\rangle \leq 0, \quad \forall z \in Q . \\
H\left(x, t, \bar{y}(x, t), \bar{u}(x, t), \psi^{0}, \psi(x, t)\right)=\max _{v \in U} H\left(x, t, \bar{y}(x, t), v, \psi^{0}, \psi(x, t)\right), \\
\text { a.e. }(x, t) \in \Omega \times[0, T] .
\end{gathered}
$$

where

$$
\begin{aligned}
H\left(x, t, y, u, \psi^{0}, \psi\right)= & \psi^{0} f^{0}(x, t, y, u)+\psi f(x, t, y, u), \\
& \forall\left(x, t, y, u, \psi^{0}, \psi\right) \in \Omega \times[0, T] \times \mathbb{R} \times U \times \mathbb{R} \times \mathbb{R} .
\end{aligned}
$$


In the above, (2.12) is called the adjoint equation, (2.13) is called the transversality condition and (2.14) is called the maximum condition. It will be seen that in the proof, we only need (2.10) holds for $\eta=\bar{y}$. Also, in $\S 5$, we will give some examples for which such a compatible condition holds.

\section{§3. Some Technical Lemmas.}

In order to derive the first order necessary conditions for optimal pairs, we need some sort of "directional derivatives" of the state $y$ and the cost functional $J(u)$ in the control variable $u$. However, since the control domain $U$ is just a metric space and there is no convexity in general, the perturbation of the control variable is restricted to be of "spike" type. Thus, to find the "directional derivative" is not obvious. In this section, we will present some technical lemmas which will give us exactly the "directional derivatives" we need in the proof of the maximum principle in $\S 4$. The results of this section is comparable with those in $[5]$ for elliptic equations (see $[13-15,20]$ also).

Lemma 3.1. Let $h^{0} \in L^{1}(\Omega)$ and $h \in L^{p}(\Omega), 1<p<\infty$. For any $\rho \in(0,1)$, we define

$$
\mathcal{E}_{\rho}=\{E \subset \Omega \mid E \text { measurable and }|E|=\rho|\Omega|\}
$$

Let the embedding $\mathcal{Y} \hookrightarrow L^{p^{\prime}}(\Omega)$ be compact $\left(p^{\prime}=\frac{p}{p-1}\right)$. Then,

$$
\inf _{E \in \mathcal{E}_{\rho}}\left\{\left|\int_{\Omega}\left(1-\frac{1}{\rho} \chi_{E_{\rho}}(x)\right) h^{0}(x) d x\right|+\left\|\left(1-\frac{1}{\rho} \chi_{E_{\rho}}\right) h\right\|_{\mathcal{Y}^{*}}\right\}=0 .
$$

Proof. Let $\rho \in(0,1)$ be given and let $\delta>0$ be arbitrary. We let $B$ be the closed unit ball in $\mathcal{Y}$. This set is compact in $L^{p^{\prime}}(\Omega)$ by our assumption. Thus, we can find a set of finitely many step functions $\Theta \equiv\left\{\theta_{i}, 1 \leq i \leq r\right\}$, such that for any $y \in B$, there exists a $\theta_{i} \in \Theta$ satisfying

$$
\left\|y-\theta_{i}\right\|_{L^{p^{\prime}}(\Omega)}<\delta
$$

Since $\Theta$ is a finite set, we may let $\left\{K_{j}\right\}_{j=1}^{m}$ be a partition of $\Omega$ with $\left|K_{j}\right|>0$ for each $1 \leq j \leq m$, such that

$$
\theta_{i}(x)=\sum_{j=1}^{m} c_{i j} \chi_{K_{j}}(x), \quad x \in \Omega \quad 1 \leq i \leq r
$$


Then, for any $y \in B$, by choosing $\theta_{i} \in \Theta$ with the property (3.3), we have

$$
\begin{aligned}
& \sum_{j=1}^{m} \int_{K_{j}}\left|y(x)-\frac{1}{\left|K_{j}\right|} \int_{K_{j}} y(\xi) d \xi\right|^{p^{\prime}} d x \\
& \leq 3^{p^{\prime}-1} \sum_{j=1}^{m}\left\{\int_{K_{j}}\left|y(x)-\theta_{i}(x)\right|^{p^{\prime}} d x+\int_{K_{j}}\left|\theta_{i}(x)-\frac{1}{\left|K_{j}\right|} \int_{K_{j}} \theta_{i}(\xi) d \xi\right|^{p^{\prime}} d x\right. \\
&\left.+\int_{K_{j}}\left|\frac{1}{\left|K_{j}\right|} \int_{K_{j}}\left(y(\xi)-\theta_{i}(\xi)\right) d \xi\right|^{p^{\prime}} d x\right\} \\
& \leq 3^{p^{\prime}-1}\left\{\int_{\Omega}\left|y(x)-\theta_{i}(x)\right|^{p^{\prime}} d x+\sum_{i=1}^{m} \frac{1}{\left|K_{j}\right|^{p^{\prime}}} \int_{K_{j}}\left|K_{j}\right|^{p^{\prime} / p} \int_{K_{j}}\left|y(\xi)-\theta_{i}(\xi)\right|^{p^{\prime}} d \xi d x\right\} \\
& \leq 3^{p^{\prime}-1}\left\{\delta^{p^{\prime}}+\int_{\Omega}\left|y(\xi)-\theta_{i}(\xi)\right|^{p^{\prime}} d \xi\right\} \leq 2 \cdot 3^{p^{\prime}-1} \delta^{p^{\prime}} .
\end{aligned}
$$

Here, we have used the fact that $\theta_{i}(x)$ is a constant on each set $K_{j}$. We have seen that the above estimate is uniform for $y \in B$.

Now, for any $y \in B$, let us define $\tilde{y}: \Omega \rightarrow \mathbb{R}$ to be the following:

$$
\widetilde{y}(x)=\frac{1}{\left|K_{j}\right|} \int_{K_{j}} y(\xi) d \xi, \quad x \in K_{j}, \quad 1 \leq j \leq m .
$$

Then, (3.5) can be written as (by setting $\varepsilon^{p^{\prime}}=2 \cdot 3^{p^{\prime}-1} \delta^{p^{\prime}}$, which is still arbitrary)

$$
\|y-\widetilde{y}\|_{L^{p^{\prime}}(\Omega)}<\varepsilon, \quad y \in B
$$

Next, on each $K_{j}$, we approximate the function $h^{0}$ and $h$ by step functions:

$$
h_{j}^{0}(x)=\sum_{i=1}^{r_{j}} \alpha_{i j} \chi_{F_{i j}}(x), \quad h_{j}(x)=\sum_{i=1}^{r_{j}} \beta_{i j} \chi_{F_{i j}}(x), \quad x \in \Omega,
$$

with $\alpha_{i j}, \beta_{i j} \in \mathbb{R},\left\{F_{i j}\right\}_{i=1}^{r_{j}}$ being a partition of $K_{j},\left|F_{i j}\right|>0$, and such that

$$
\int_{K_{j}}\left|h^{0}(x)-h_{j}^{0}(x)\right| d x+\int_{K_{j}}\left|h(x)-h_{j}(x)\right| d x<\varepsilon\left|K_{j}\right|, \quad 1 \leq j \leq m .
$$

Let us take $E_{\rho}^{i j} \subset F_{i j}$, such that $\left|E_{\rho}^{i j}\right|=\rho\left|F_{i j}\right|$. Set $E_{\rho}^{i}=\bigcup_{i=1}^{r_{j}} E_{\rho}^{i j}$. Since $h_{j}^{0}(x)$ and $h_{j}(x)$ are simple functions, we have

$$
\left\{\begin{array}{l}
\int_{K_{j}} h_{j}^{0}(x) d x=\int_{K_{j}} \frac{1}{\rho} \chi_{E_{\rho}^{j}}(x) h_{j}^{0}(x) d x \\
\int_{K_{j}} h_{j}(x) d x=\int_{K_{j}} \frac{1}{\rho} \chi_{E_{\rho}^{j}}(x) h_{j}(x) d x,
\end{array}\right.
$$


Finally, we take $E_{\rho}=\bigcup_{j=1}^{m} E_{\rho}^{j}$. Then, $E_{\rho} \in \mathcal{E}_{\rho}$, and for any $y \in B$,

$$
\begin{aligned}
& \left|\int_{\Omega}\left(1-\frac{1}{\rho} \chi_{E_{\rho}}\right) h(x) y(x) d x\right| \leq\left|\int_{\Omega}\left(1-\frac{1}{\rho} \chi_{E_{\rho}}\right) h(x) \widetilde{y}(x) d x\right| \\
& \quad+\int_{\Omega}\left(1+\frac{1}{\rho} \chi_{E_{\rho}}\right)|h(x)||y(x)-\tilde{y}(x)| d x \\
& \leq\left|\sum_{j=1}^{m} \int_{K_{j}}\left(1-\frac{1}{\rho} \chi_{E_{\rho}^{j}}(x)\right) h(x) \tilde{y}(x) d x\right|+\left(1+\frac{1}{\rho}\right)\|h\|_{L^{p}(\Omega)}|| y-\tilde{y} \|_{L^{p^{\prime}}(\Omega)} .
\end{aligned}
$$

From (3.7) we see that

$$
\left(1+\frac{1}{\rho}\right)\|h\|_{L^{p}(\Omega)}\|y-\widetilde{y}\|_{L^{p^{\prime}}(\Omega)} \leq \varepsilon\left(1+\frac{1}{\rho}\right)\|h\|_{L^{p}(\Omega)} .
$$

On the other hand, we notice that $\widetilde{y}(x)$ is a constant on each set $K_{j}$ (we denote this constant by $\left.\widetilde{y}\left(K_{j}\right)\right)$. Thus, by (3.9)-(3.10) and (3.6), we have

$$
\begin{aligned}
& \left|\sum_{j=1}^{m} \int_{K_{j}}\left(1-\frac{1}{\rho} \chi_{E_{\rho}^{j}}(x)\right) h(x) \widetilde{y}(x) d x\right| \\
& \leq \sum_{j=1}^{m}\left|\widetilde{y}\left(K_{j}\right)\right| \int_{K_{j}}\left(1+\frac{1}{\rho} \chi_{E_{\rho}^{j}}(x)\right)\left|h(x)-h_{j}(x)\right| d x \\
& \quad+\left|\sum_{j=1}^{m} \widetilde{y}\left(K_{j}\right) \int_{K_{j}}\left(1-\frac{1}{\rho} \chi_{E_{\rho}^{j}}\right) h_{j}(x) d x\right| \\
& \leq\left(1+\frac{1}{\rho}\right) \sum_{j=1}^{m} \varepsilon\left|K_{j}\right|\left|\widetilde{y}\left(K_{j}\right)\right| \leq \varepsilon\left(1+\frac{1}{\rho}\right)\|y\|_{L^{1}(\Omega)} \leq \varepsilon\left(1+\frac{1}{\rho}\right) C .
\end{aligned}
$$

Here, $\|y\|_{L^{1}(\Omega)} \leq C\|y\| y \leq C$, since $y \in B$. Thus, (3.11)-(3.13) imply that

$$
\left\|\left(1-\frac{1}{\rho} \chi_{E \rho}\right) h\right\|_{\mathcal{Y}^{*}} \leq \varepsilon\left(1+\frac{1}{\rho}\right)\left(C+\|h\|_{L^{p}(\Omega)}\right) .
$$

On the other hand by (3.9)-(3.10) again, we have

$$
\begin{aligned}
& \left|\int_{\Omega}\left(1-\frac{1}{\rho} \chi_{E_{\rho}}(x)\right) h^{0}(x) d x\right| \leq \sum_{j=1}^{m}\left|\int_{K_{j}}\left(1-\frac{1}{\rho} \chi_{E_{\rho}^{j}}(x)\right) h^{0}(x) d x\right| \\
& \leq \sum_{j=1}^{m}\left(1+\frac{1}{\rho}\right) \int_{K_{j}}\left|h^{0}(x)-h_{j}^{0}(x)\right| d x+\sum_{j=1}^{m}\left|\int_{K_{j}}\left(1-\frac{1}{\rho} \chi_{E_{\rho}^{j}}\right) h_{j}^{0}(x) d x\right| \\
& <\varepsilon\left(1+\frac{1}{\rho}\right) .
\end{aligned}
$$


Therefore, our conclusion follows.

The above result was proved for $\mathcal{Y}=W_{0}^{1, p}(\Omega)$ in $[5]$ using different methods. The proof given here is inspired by a personal communication of the second author with E. Casas.

Now we consider the equation

$$
\left\{\begin{array}{l}
\zeta_{t}-\left(a_{i j}(x, t) \zeta_{x_{i}}\right)_{x_{j}}+c_{\rho}(x, t) \zeta(x, t)=\left(1-\frac{1}{\rho} \chi_{E_{\rho}}(x, t)\right) h(x, t), \text { in } \Omega_{T} \\
\left.\zeta\right|_{\partial_{p} \Omega_{T}}=0 .
\end{array}\right.
$$

where $a_{i j}$ satisfies (A1) and $\partial_{p} \Omega_{T}=(\partial \Omega \times[0, T]) \bigcup(\bar{\Omega} \times\{0\})$ is the parabolic boundary of $\Omega_{T}$. It is clear that the solution $\zeta=\zeta_{E_{\rho}}(x, t)$ is uniquely determined by the choice of the coefficient $c_{\rho}$ and the set $E_{\rho}$.

Lemma 3.2. Suppose that $\frac{n+2}{2}<p<\infty$. Then, there exists a $\theta \in(0,1)$ such that for each $h^{0} \in L^{1}\left(\Omega_{T}\right), h \in L^{p}\left(\Omega_{T}\right), \mathcal{K}=\left\{c(x, t) ;\|c\|_{L^{\infty}\left(\Omega_{T}\right)} \leq K\right\}(K>0)$, and any $\rho \in(0,1)$,

$$
\inf _{E_{\rho} \in \mathcal{E}_{\rho}} \sup _{c_{\rho} \in \mathcal{K}}\left\{\left|\int_{\Omega_{T}}\left(1-\frac{1}{\rho} \chi_{E_{\rho}}(x, t)\right) h^{0}(x, t) d x d t\right|+\left\|\zeta_{E_{\rho}}\right\|_{C^{\theta, \theta / 2}\left(\bar{\Omega}_{T}\right)}\right\}=0 .
$$

Proof. By the assumption, $h \in L^{p}\left(\Omega_{T}\right), p>\frac{n+2}{2}$. Therefore the right-hand-side of the equation (3.16) is in $L^{p}\left(\Omega_{T}\right)$, (although the $L^{p}\left(\Omega_{T}\right)$ norm may blow up as $\rho \rightarrow 0$ ). It follows from the parabolic estimates [12, Chapter III, section 10] that there exists $\beta \in(0,1)$, such that

$$
\sup _{E_{\rho} \in \mathcal{E}_{\rho}} \sup _{c_{\rho} \in \mathcal{K}}\left\|\zeta_{E_{\rho}}\right\|_{C^{\beta, \beta / 2}\left(\Omega_{T}\right)} \leq C_{\rho}
$$

We claim that, for any $0<\theta<\beta$, (3.17) hods. In fact, we first note that the identity mapping $C^{\beta, \beta / 2}\left(\bar{\Omega}_{T}\right) \hookrightarrow L^{p^{\prime}}\left(\Omega_{T}\right)$ is compact. Therefore if we take $\mathcal{Y}=C^{\beta, \beta / 2}\left(\bar{\Omega}_{T}\right)$ in Lemma 3.1, then

$$
\begin{aligned}
& \inf _{E_{\rho} \in \mathcal{E}_{\rho}} \sup _{c_{\rho} \in \mathcal{K}}\left\{\left|\int_{\Omega_{T}}\left(1-\frac{1}{\rho} \chi_{E_{\rho}}\right) h^{0} d x d t\right|+\left|\int_{\Omega_{T}}\left(1-\frac{1}{\rho} \chi_{E_{\rho}}\right) h \cdot \zeta_{E_{\rho}} d x d t\right|\right\} \\
& \leq \inf _{E_{\rho} \in \mathcal{E}_{\rho}}\left\{\left|\int_{\Omega_{T}}\left(1-\frac{1}{\rho} \chi_{E_{\rho}}\right) h^{0} d x d t\right|+C_{\rho} \|\left.\left(1-\frac{1}{\rho} \chi_{E_{\rho}}\right) h\right|_{\mathcal{Y}^{*}}\right\}=0 .
\end{aligned}
$$


Using a change of variable $\zeta(x, t)=\phi(x, t) e^{K t}$ if necessary, we may assume without loss of generality that $c_{\rho}(x, t) \geq 0$. Multiplying equation (3.16) with $\zeta_{E_{\rho}}$ and integrating over $\Omega_{T}$, we immediately obtain

$$
\begin{array}{r}
\int_{\Omega}\left(\zeta_{E_{\rho}}\right)^{2}(x, T) d x+\int_{\Omega_{T}}\left|\nabla \zeta_{E_{\rho}}(x, \tau)\right|^{2} d x d \tau \\
\leq C\left|\int_{0}^{T} \int_{\Omega}\left(1-\frac{1}{\rho} \chi_{E_{\rho}}\right) h \cdot \zeta_{E_{\rho}} d x d t\right|
\end{array}
$$

Notice that $\zeta_{E_{\rho}}=0$ on $\partial \Omega \times\{t\}$, for each $t \in(0, T)$. Therefore, $\int_{\Omega}\left(\zeta_{E_{\rho}}\right)^{2}(x, t) d x \leq$ $C \int_{\Omega}\left|\nabla \zeta_{E_{\rho}}\right|^{2}(x, t) d x$, by Poincaré's inequality. Integrating over $t \in[0, T]$, and taking (3.20) into account, yields

$$
\int_{\Omega_{T}}\left(\zeta_{E_{\rho}}\right)^{2}(x, t) d x d t \leq C\left|\int_{\Omega_{T}}\left(1-\frac{1}{\rho} \chi_{E_{\rho}}(x, t)\right) h(x, t) \zeta_{E_{\rho}}(x, t) d x d t\right| .
$$

By the interpolation theorem (see Lemma 3.4 below), for any $\varepsilon>0$, there exists $C_{\varepsilon}>0$, such that

$$
\|\zeta\|_{C^{\theta, \theta / 2}\left(\bar{\Omega}_{T}\right)} \leq \varepsilon\|\zeta\|_{C^{\beta, \beta / 2}\left(\bar{\Omega}_{T}\right)}+C_{\varepsilon}\|\zeta\|_{L^{2}\left(\Omega_{T}\right)}, \quad \forall \zeta \in C^{\beta, \beta / 2}\left(\bar{\Omega}_{T}\right)
$$

Using (3.19), (3.21) and (3.22), we obtain

$$
\inf _{E \in \mathcal{E}_{\rho}} \sup _{c_{\rho} \in \mathcal{K}}\left\{\left|\int_{\Omega_{T}}\left(1-\frac{1}{\rho} \chi_{E_{\rho}}(x, t)\right) h^{0}(x, t) d x d t\right|+\left\|\zeta_{E_{\rho}}\right\|_{C^{\theta, \theta / 2}\left(\bar{\Omega}_{T}\right)}\right\} \leq \varepsilon C_{\rho} .
$$

Since $\varepsilon$ can be arbitrarily small, the lemma follows.

Now, for any feasible pair $(y, u)$, we define

$$
\left\{\begin{array}{l}
c(x, t)=-f_{y}(x, t, y(x, t), u(x, t)) \\
c^{0}(x, t)=f_{y}^{0}(x, t, y(x, t), u(x, t))
\end{array}\right.
$$

and for given $v \in \mathcal{U}$,

$$
\left\{\begin{array}{l}
h(x, t)=f(x, t, y(x, t), v(x, t))-f(x, t, y(x, t), u(x, t)) \\
h^{0}(x, t)=f^{0}(x, t, y(x, t), v(x, t))-f^{0}(x, t, y(x, t), u(x, t))
\end{array}\right.
$$


Consider the following problem

$$
\left\{\begin{array}{l}
z_{t}-\sum_{i, j=1}^{n}\left(a_{i j}(x, t) z_{x_{i}}\right)_{x_{j}}+c(x, t) z=h(x, t), \quad \text { in } \Omega_{T} \\
\left.z\right|_{\partial_{p} \Omega_{T}}=0 .
\end{array}\right.
$$

Clearly, since $h \in L^{\infty}\left(\Omega_{T}\right)$, this problem admits a unique solution $z \in C^{\beta, \beta / 2}\left(\bar{\Omega}_{T}\right) \bigcap L^{2}(0$, $T ; H_{0}^{1}(\Omega)$ ), as in Proposition 2.1 .

Our main result of this section is the following.

Theorem 3.3. Let $(y, u)$ be a given feasible pair and $v \in \mathcal{U}$ be fixed. Then, for any $\rho \in(0,1)$, there exists a measurable set $E_{\rho} \subset \Omega_{T}$, with property $\left|E_{\rho}\right|=\rho\left|\Omega_{T}\right|$, such that if we define $u_{\rho}$ by

$$
u_{\rho}(x, t)= \begin{cases}u(x, t), & \text { if }(x, t) \in \Omega_{T} \backslash E_{\rho} \\ v(x, t), & \text { if }(x, t) \in E_{\rho}\end{cases}
$$

and let $y_{\rho}$ be the state corresponding to $u_{\rho}$, then the following hold:

$$
\left\{\begin{array}{l}
y_{\rho}=y+\rho z+r_{\rho} \\
\lim _{\rho \rightarrow 0} \frac{1}{\rho}\left\|r_{\rho}\right\|_{C^{\theta, \theta / 2}\left(\bar{\Omega}_{T}\right)}=0
\end{array}\right.
$$

for some $\theta \in(0,1)$, and

$$
\left\{\begin{array}{l}
J\left(u_{\rho}\right)=J(u)+\rho z^{0}+r_{\rho}^{0} \\
\lim _{\rho \rightarrow 0} \frac{1}{\rho}\left|r_{\rho}^{0}\right|=0
\end{array}\right.
$$

where $z$ is the solution of (3.25) and $z^{0}$ is given by

$$
z^{0}=\int_{\Omega}\left[c^{0}(x, t) z(x, t)+h^{0}(x, t)\right] d x
$$

Proof. First, we recall the so-called Ekeland distance. For any $u, v \in \mathcal{U}$, we let

$$
\bar{d}(u, v)=|\{(x, t) \in \Omega \mid u(x, t) \neq v(x, t)\}| .
$$

It is standard that $(\mathcal{U}, \bar{d}(\cdot, \cdot))$ is a complete metric space (see $[8])$. Clearly, $\bar{d}(u, v) \leq\left|E_{\rho}\right|$. 
Now, we set

$$
z_{\rho}(x, t)=\frac{y_{\rho}(x, t)-y(x, t)}{\rho}, \quad x \in \Omega .
$$

Then, $z_{\rho}$ satisfies the following

$$
\left\{\begin{array}{l}
\left(z_{\rho}\right)_{t}-\sum_{i, j=1}^{n}\left(a_{i j}(x, t)\left(z_{\rho}\right)_{x_{i}}\right)_{x_{j}}+c_{\rho}(x, t) z_{\rho}=\frac{1}{\rho} \chi_{E_{\rho}}(x, t) h(x, t), \\
\left.z_{\rho}\right|_{\partial_{p} \Omega_{T}}=0
\end{array}\right.
$$

where

$$
c_{\rho}(x, t)=-\int_{0}^{1} f_{y}\left(x, t, y(x, t)+\tau\left(y_{\rho}(x, t)-y(x, t)\right), u_{\rho}(x, t)\right) d \tau .
$$

We see that (note (2.9) and $(2.3)), c_{\rho}(x, t)$ and $h(x, t)$ are uniformly bounded (with the bounds independent of $E_{\rho}$, the controls $u$ and $\left.v\right)$. The function $h(x, t)$ is actually independent of the set $E_{\rho}$; we shall use this fact when we apply Lemma 3.2. Since $h \in L^{\infty}\left(\Omega_{T}\right) \subset L^{p}\left(\Omega_{T}\right)$ for any $p>1$, the parabolic Hölder's estimates implies that $y_{\rho}-y=\rho z_{\rho}$ satisfies, for fixed $p>\frac{n+2}{2}$,

$$
\left\|y_{\rho}-y\right\|_{C^{\beta, \beta / 2}\left(\bar{\Omega}_{T}\right)} \leq C\left\|\chi_{E_{\rho}}\right\|_{L^{p}\left(\Omega_{T}\right)} \equiv \omega(C \rho) \rightarrow 0, \quad \text { as } \rho \rightarrow 0,
$$

where the constant $C$ is independent of $E_{\rho}$, and $\omega$ is a modulus of continuity. It follows that

$$
c_{\rho}(x, t) \rightarrow c(x, t) \equiv-f_{y}(x, t, y(x, t), u(x, t)), \quad \text { in } L^{p}\left(\Omega_{T}\right), 1 \leq p<\infty
$$

By recalling $z$, the solution of $(3.25)$, we have the following:

$$
\left\{\begin{array}{l}
\left(z_{\rho}-z\right)_{t}-\sum_{i, j=1}^{n}\left(a_{i j}(x, t)\left(z_{\rho}-z\right)_{x_{i}}\right)_{x_{j}}+c_{\rho}(x, t)\left(z_{\rho}-z\right) \\
\quad=-\left(c_{\rho}(x, t)-c(x, t)\right) z-\left(1-\frac{1}{\rho} \chi_{E_{\rho}}(x, t)\right) h(x, t), \\
\left.\left(z_{\rho}-z\right)\right|_{\partial_{p} \Omega_{T}}=0,
\end{array}\right.
$$

We note that the above equation is linear in $\left(z_{\rho}-z\right)$. Thus, we may write $z_{\rho}-z=\bar{\zeta}_{\rho}+\zeta_{\rho}$ with $\bar{\zeta}_{\rho}$ and $\zeta_{\rho}$ satisfying the following:

$$
\left\{\begin{array}{l}
\left(\bar{\zeta}_{\rho}\right)_{t}-\sum_{i, j=1}^{n}\left(a_{i j}(x, t)\left(\bar{\zeta}_{\rho}\right)_{x_{i}}\right)_{x_{j}}+c_{\rho}(x, t) \bar{\zeta}_{\rho}=-\left(c_{\rho}(x, t)-c(x, t)\right) z \\
\left.\bar{\zeta}_{\rho}\right|_{\partial_{p} \Omega_{T}}=0
\end{array}\right.
$$


and

$$
\left\{\begin{array}{l}
\left(\zeta_{\rho}\right)_{t}-\sum_{i, j=1}^{n}\left(a_{i j}(x, t)\left(\zeta_{\rho}\right)_{x_{i}}\right)_{x_{j}}+c_{\rho}(x, t) \zeta_{\rho}=-\left(1-\frac{1}{\rho} \chi_{E_{\rho}}(x, t)\right) h(x, t), \\
\left.\zeta_{\rho}\right|_{\partial_{p} \Omega_{T}}=0
\end{array}\right.
$$

By Hölder estimates again, (notice that $z \in C^{\beta, \beta / 2}\left(\bar{\Omega}_{T}\right) \subset L^{\infty}\left(\Omega_{T}\right)$, and $p$ is fixed with $\left.p>\frac{n+2}{2}\right)$, we have

$$
\left\|\bar{\zeta}_{\rho}\right\|_{C^{\beta, \beta / 2}\left(\bar{\Omega}_{T}\right)} \leq C\left\|\left(c_{\rho}-c\right) z\right\|_{L^{p}\left(\Omega_{T}\right)}=o(1) \quad \text { as } \rho \rightarrow 0
$$

all the constants involved in the above are independent of the choices of $E_{\rho}$.

Now we fix $\theta \in(0, \beta)$ as in Lemma 3.2. Then we can choose $E_{\rho} \subset \Omega_{T}$ with the property $\left|E_{\rho}\right|=\rho\left|\Omega_{T}\right|$, such that the solution $\zeta_{\rho}$ of (3.38) satisfies

$$
\left|\int_{\Omega_{T}}\left(1-\frac{1}{\rho} \chi_{E_{\rho}}(x, t)\right) h^{0}(x, t) d x d t\right|+\left\|\zeta_{\rho}\right\|_{C^{\theta, \theta / 2}\left(\bar{\Omega}_{T}\right)} \leq \rho .
$$

This proves (3.27). The proof of (3.28) is similar but simpler.

The above result will play a very important role in the proof of our main result (Theorem 2.2). Conclusion (3.27) gives a "Taylor expansion" (of first order) in the space $C^{\theta, \theta / 2}\left(\bar{\Omega}_{T}\right) \subset C\left(\bar{\Omega}_{T}\right)$. This will be sufficient for us to deal with the pointwise state constraint. It is not hard for us to see that the stronger the topology under which (3.27) holds, the harder for us to prove it. Thus, for example, if in $(3.27), C^{\theta, \theta / 2}\left(\bar{\Omega}_{T}\right)$ is replaced by $L^{p}\left(\Omega_{T}\right)$, then it will be much easier to prove it. In another word, an $L^{p}\left(\Omega_{T}\right)$ constraint of the state is much easier to treat than a $C\left(\bar{\Omega}_{T}\right)$ constraint.

We now give a proof for the interpolation theorem used in the proof of Lemma 3.2. The identity mappings $C^{\beta, \beta / 2}\left(\bar{\Omega}_{T}\right) \hookrightarrow C^{\theta, \theta / 2}\left(\bar{\Omega}_{T}\right) \hookrightarrow L^{2}\left(\Omega_{T}\right)$ are continuous and compact. Therefore the interpolation follows from a compactness argument. However, the compactness argument does not give us the exact form of the the constants $C_{\varepsilon}$. Lemma 3.4 below is a stronger statement.

The interpolation involves different type of spaces. Nonetheless, the proof is similar to that in, for example, [11]. 
Lemma 3.4. Suppose that $\partial \Omega$ is Lipschitz continuous, $0 \leq \theta<\beta<1$ and $0<p \leq \infty$. Then there exists a constant $C$, depending only on $\Omega$ and $T$, such that

$$
\begin{gathered}
\|\zeta\|_{C^{\theta, \theta / 2}\left(\bar{\Omega}_{T}\right)} \leq 4 \varepsilon[\zeta]_{C^{\beta, \beta / 2}\left(\bar{\Omega}_{T}\right)}+\frac{3 C^{1 / p}}{\varepsilon^{\mu}}\|\zeta\|_{L^{p}\left(\Omega_{T}\right)}, \\
\forall \zeta \in C^{\beta, \beta / 2}\left(\bar{\Omega}_{T}\right), \quad \forall 0<\varepsilon \leq 1
\end{gathered}
$$

where $\mu=\frac{\theta}{(\beta-\theta)}+\frac{n+2}{(\beta-\theta) p}$, and

$$
\left\{\begin{array}{l}
{[\zeta]_{C^{\theta, \theta / 2}\left(\bar{\Omega}_{T}\right)}=\sup \left\{\frac{|\zeta(x, t)-\zeta(\bar{x}, \bar{t})|}{\left(\sqrt{|x-\bar{x}|^{2}+|t-\bar{t}|}\right)^{\theta}} ; \quad(x, t) \neq(\bar{x}, \bar{t}) \in \bar{\Omega}_{T}\right\}} \\
\|\zeta\|_{C^{\theta, \theta / 2}\left(\bar{\Omega}_{T}\right)}=\|\zeta\|_{C\left(\bar{\Omega}_{T}\right)}+[\zeta]_{C^{\theta, \theta / 2}\left(\bar{\Omega}_{T}\right)} \quad \text { when } 0<\theta<1, \\
\|\zeta\|_{C^{\theta, \theta / 2}\left(\bar{\Omega}_{T}\right)}=\|\zeta\|_{C\left(\bar{\Omega}_{T}\right)} \quad \text { when } \theta=0 .
\end{array}\right.
$$

(The $\|\cdot\|_{L^{p}\left(\Omega_{T}\right)}$ should be understood in the usual sense, it should be noted that it is not a norm when $0<p<1$.)

Proof. We let $\delta=\varepsilon^{1 /(\beta-\theta)}$, then $0<\delta \leq 1$. Splitting the sup in (3.43) into two set $\left\{\sqrt{|x-\bar{x}|^{2}+|t-\bar{t}|} \leq \delta\right\}$ and $\left\{\sqrt{|x-\bar{x}|^{2}+|t-\bar{t}|}>\delta\right\}$ immediately gives us

$$
\|\zeta\|_{C^{\theta, \theta / 2}\left(\bar{\Omega}_{T}\right)} \leq \delta^{\beta-\theta}[\zeta]_{C^{\beta, \beta / 2}\left(\bar{\Omega}_{T}\right)}+\left(1+\frac{2}{\delta^{\theta}}\right)\|\zeta\|_{C\left(\bar{\Omega}_{T}\right)}
$$

from which the case $p=\infty$ follows.

Now consider the case $0<p<\infty$. Since $\zeta$ is continuous on $\bar{\Omega}_{T},\|\zeta\|_{C\left(\bar{\Omega}_{T}\right)}=|\zeta(\tilde{x}, \tilde{t})|$, for some $(\tilde{x}, \tilde{t}) \in \bar{\Omega}_{T}$. Now let $B_{\delta}=\left\{(x, t) \in \bar{\Omega}_{T} ; \quad \sqrt{|x-\tilde{x}|^{2}+|t-\widetilde{t}|} \leq \delta\right\}$, then by the mean value Theorem,

$$
\left(\frac{1}{\left|B_{\delta} \bigcap \bar{\Omega}_{T}\right|} \int_{B_{\delta} \bigcap \bar{\Omega}_{T}}|\zeta(x, t)|^{p} d x d t\right)^{1 / p}=\left|\zeta\left(x^{*}, t^{*}\right)\right|
$$

for some $\left(x^{*}, t^{*}\right) \in B_{\delta} \bigcap \bar{\Omega}_{T}$. Since $\partial \Omega$ is Lipschitz continuous, $\left|B_{\delta} \bigcap \bar{\Omega}_{T}\right| \geq \delta^{n+2} / C$ for some generic constant $C>0$. It follows that

$$
\begin{aligned}
\|\zeta\|_{C\left(\bar{\Omega}_{T}\right)}=|\zeta(\widetilde{x}, \tilde{t})| & \leq\left|\zeta(\widetilde{x}, \widetilde{t})-\zeta\left(x^{*}, t^{*}\right)\right|+\left|\zeta\left(x^{*}, t^{*}\right)\right| \\
& \leq \delta^{\beta}[\zeta]_{C^{\beta, \beta / 2}\left(\bar{\Omega}_{T}\right)}+\left(\frac{C}{\delta^{n+2}}\right)^{1 / p}\|\zeta\|_{L^{p}\left(\Omega_{T}\right)}
\end{aligned}
$$


the case $\theta=0$ follows immediately. Now substituting (3.45) into (3.44), we obtain

$$
\|\zeta\|_{C^{\theta, \theta / 2}\left(\bar{\Omega}_{T}\right)} \leq 4 \delta^{\beta-\theta}[\zeta]_{C^{\beta, \beta / 2}\left(\bar{\Omega}_{T}\right)}+\frac{3}{\delta^{\theta}}\left(\frac{C}{\delta^{n+2}}\right)^{1 / p}\|\zeta\|_{L^{p}\left(\Omega_{T}\right)}
$$

and the general case $0<\theta<\beta<1,0<p<\infty$ follows.

\section{$\S 4$. Proof of Theorem 2.2.}

In this section, we are going to prove our main theorem of this paper.

Proof of Theorem 2.2. Let $(\bar{y}, \bar{u})$ be an optimal pair. For any $u \in \mathcal{U}$, let $y(\cdot, \cdot ; u)$ be the corresponding state, emphasizing the dependence of it on the control. For any $\varepsilon>0$, we define

$$
J_{\varepsilon}(u)=\left\{\left[(J(u)-J(\bar{u})+\varepsilon)^{+}\right]^{2}+d_{Q}(G(y(\cdot, \cdot ; u)))^{2}\right\}^{1 / 2} .
$$

Clearly, this functional is continuous on the (complete) metric space $(\mathcal{U}, \bar{d})$ (recall that $\bar{d}$ is the Ekeland distance, see (3.30)). Also, we have

$$
\begin{gathered}
J_{\varepsilon}(u)>0, \quad \forall u \in \mathcal{U}, \\
J_{\varepsilon}(\bar{u})=\varepsilon \leq \inf _{\mathcal{U}} J_{\varepsilon}(u)+\varepsilon .
\end{gathered}
$$

Hence, by Ekeland's variational principle $([6])$, we can find a $u^{\varepsilon} \in \mathcal{U}$, such that

$$
\begin{gathered}
\bar{d}\left(\bar{u}, u^{\varepsilon}\right) \leq \sqrt{\varepsilon}, \\
J_{\varepsilon}\left(u^{\varepsilon}\right) \leq J_{\varepsilon}(\bar{u}), \\
J_{\varepsilon}(\widehat{u})-J_{\varepsilon}\left(u^{\varepsilon}\right) \geq-\sqrt{\varepsilon} d\left(\widehat{u}, u^{\varepsilon}\right), \quad \forall \widehat{u} \in \mathcal{U} .
\end{gathered}
$$

We let $v \in \mathcal{U}$ and $\varepsilon>0$ be fixed and let $y^{\varepsilon}=y\left(\cdot, \cdot ; u^{\varepsilon}\right)$. By Theorem 3.3, we know that for any $\rho \in(0,1)$, there exists a measurable set $E_{\rho}^{\varepsilon} \subset \Omega_{T}$ with the property $\left|E_{\rho}^{\varepsilon}\right|=\rho\left|\Omega_{T}\right|$, such that if we define

$$
u_{\rho}^{\varepsilon}(x, t)= \begin{cases}u^{\varepsilon}(x, t), & \text { if }(x, t) \in \Omega_{T} \backslash E_{\rho}^{\varepsilon} \\ v(x, t), & \text { if }(x, t) \in E_{\rho}^{\varepsilon}\end{cases}
$$


and let $y_{\rho}^{\varepsilon}=y\left(\cdot, \cdot ; u_{\rho}^{\varepsilon}\right)$ be the corresponding state, then

$$
\left\{\begin{array}{l}
y_{\rho}^{\varepsilon}=y^{\varepsilon}+\rho z^{\varepsilon}+r_{\rho}^{\varepsilon}, \\
J\left(u_{\rho}^{\varepsilon}\right)=J\left(u^{\varepsilon}\right)+\rho z^{0, \varepsilon}+r_{\rho}^{0, \varepsilon},
\end{array}\right.
$$

where $z^{\varepsilon}$ and $z^{0, \varepsilon}$ satisfy the following:

$$
\left\{\begin{array}{l}
z_{t}^{\varepsilon}-\sum_{i, j}^{n}\left(a_{i j}(x, t) z_{x_{i}}^{\varepsilon}\right)_{x_{j}}-f_{y}\left(x, t, y^{\varepsilon}(x, t), u^{\varepsilon}(x, t)\right) z^{\varepsilon}=h^{\varepsilon}(x, t), \quad \text { in } \Omega_{T}, \\
\left.z\right|_{\partial_{p} \Omega_{T}}=0 .
\end{array}\right.
$$

$$
z^{0, \varepsilon}=\int_{\Omega_{T}}\left[f_{y}^{0}\left(x, t, y^{\varepsilon}(x, t), u^{\varepsilon}(x, t)\right) z^{\varepsilon}(x, t)+h^{0, \varepsilon}(x, t)\right] d x
$$

with

$$
\left\{\begin{array}{l}
h^{\varepsilon}(x, t)=f\left(x, t, y^{\varepsilon}(x, t), v(x, t)\right)-f\left(x, t, y^{\varepsilon}(x, t), u^{\varepsilon}(x, t)\right), \\
h^{0, \varepsilon}(x, t)=f^{0}\left(x, t, y^{\varepsilon}(x, t), v(x, t)\right)-f^{0}\left(x, t, y^{\varepsilon}(x, t), u^{\varepsilon}(x, t)\right) .
\end{array}\right.
$$

and for some $\theta \in(0,1)$,

$$
\lim _{\rho \rightarrow 0} \frac{1}{\rho}\left\|r_{\rho}^{\varepsilon}\right\|_{C^{\theta, \theta / 2}\left(\bar{\Omega}_{T}\right)}=\lim _{\rho \rightarrow 0} \frac{1}{\rho}\left|r_{\rho}^{0, \varepsilon}\right|=0 .
$$

Now, we take $\widehat{u}=u_{\rho}^{\varepsilon}$ in (4.6). Then, it follows that

$$
\begin{aligned}
& -\sqrt{\varepsilon}\left|\Omega_{T}\right| \leq \frac{J_{\varepsilon}\left(u_{\rho}^{\varepsilon}\right)-J_{\varepsilon}\left(u^{\varepsilon}\right)}{\rho} \\
& =\frac{1}{J_{\varepsilon}\left(u_{\rho}^{\varepsilon}\right)+J_{\varepsilon}\left(u^{\varepsilon}\right)}\left\{\frac{\left[\left(J\left(u_{\rho}^{\varepsilon}\right)-J(\bar{u})+\varepsilon\right)^{+}\right]^{2}-\left[\left(J\left(u^{\varepsilon}\right)-J(\bar{u})+\varepsilon\right)^{+}\right]^{2}}{\rho}\right. \\
& \left.\quad+\frac{d_{Q}\left(G\left(y_{\rho}^{\varepsilon}\right)\right)^{2}-d_{Q}\left(G\left(y^{\varepsilon}\right)\right)^{2}}{\rho}\right\} \\
& \rightarrow \frac{\left(J\left(u^{\varepsilon}\right)-J(\bar{u})+\varepsilon\right)^{+}}{J_{\varepsilon}\left(u^{\varepsilon}\right)} z^{0, \varepsilon}+\left\langle\frac{d_{Q}\left(G\left(y^{\varepsilon}\right)\right) \xi_{\varepsilon}}{J_{\varepsilon}\left(u^{\varepsilon}\right)}, G^{\prime}\left(y^{\varepsilon}\right) z^{\varepsilon}\right\rangle, \quad(\rho \rightarrow 0),
\end{aligned}
$$

where

$$
\xi_{\varepsilon}= \begin{cases}\nabla d_{Q}\left(G\left(y^{\varepsilon}\right)\right), & \text { if } G\left(y^{\varepsilon}\right) \notin Q, \\ 0, & \text { if } G\left(y^{\varepsilon}\right) \in Q .\end{cases}
$$


We note that since $G: C_{0}\left(\bar{\Omega}_{T}\right) \rightarrow Z$, to obtain the convergence in (4.13), the expansion (4.8) in the space $C_{0}\left(\bar{\Omega}_{T}\right)$ is necessary.

Next, we define $\left(\varphi^{0, \varepsilon}, \varphi^{\varepsilon}\right) \in[0,1] \times \mathcal{M}\left(\bar{\Omega}_{T}\right)$ as follows:

$$
\left\{\begin{array}{l}
\varphi^{0, \varepsilon}=\frac{\left(J\left(u^{\varepsilon}\right)-J(\bar{u})+\varepsilon\right)^{+}}{J_{\varepsilon}\left(u^{\varepsilon}\right)} \\
\varphi^{\varepsilon}=\frac{d_{Q}\left(G\left(y^{\varepsilon}\right)\right) \xi_{\varepsilon}}{J_{\varepsilon}\left(u^{\varepsilon}\right)}
\end{array}\right.
$$

Then we see that (4.13) becomes

$$
-\sqrt{\varepsilon}\left|\Omega_{T}\right| \leq \varphi^{0, \varepsilon} z^{0, \varepsilon}+\left\langle\varphi^{\varepsilon}, G^{\prime}\left(y^{\varepsilon}\right) z^{\varepsilon}\right\rangle
$$

By (2.8) and (4.1), we have

$$
\left|\varphi^{0, \varepsilon}\right|^{2}+\left\|\varphi^{\varepsilon}\right\|_{Z^{*}}^{2}=1 \text {. }
$$

On the other hand, by the definition of subdifferential, we have

$$
\left\langle\varphi^{\varepsilon}, \eta-G\left(y^{\varepsilon}\right)\right\rangle \leq 0, \quad \forall \eta \in Q
$$

Next, by (4.4), we have

$$
\left\|y^{\varepsilon}-\bar{y}\right\|_{C^{\beta, \beta / 2}\left(\bar{\Omega}_{T}\right)} \rightarrow 0, \quad(\varepsilon \rightarrow 0) .
$$

Thus, (4.18) implies

$$
\left\langle\varphi^{\varepsilon}, \eta-G(\bar{y})\right\rangle \leq\left\langle\varphi^{\varepsilon}, G\left(y^{\varepsilon}\right)-G(\bar{y})\right\rangle \leq\left\|G\left(y^{\varepsilon}\right)-G(\bar{y})\right\|_{Z} \rightarrow 0, \quad \forall \eta \in Q
$$

Since, $Q$ is finite codimensional in $Z$, from [15, Lemma 3.2], we know that by extracting some subsequence, still denoted by itself, one has

$$
\left(\varphi^{0, \varepsilon}, \varphi^{\varepsilon}\right) \stackrel{*}{\rightarrow}\left(\varphi^{0, \varepsilon}, \varphi\right) \neq 0
$$

On the other hand, from (4.19) and the equations (4.9) (4.10), we have

$$
\left\{\begin{array}{l}
z^{\varepsilon} \rightarrow z, \quad \text { in } C^{\beta, \beta / 2}\left(\bar{\Omega}_{T}\right), \quad(\varepsilon \rightarrow 0), \\
z^{0, \varepsilon} \rightarrow z^{0}
\end{array}\right.
$$


where $z$ is the solution of the following variational system:

$$
\left\{\begin{array}{l}
z_{t}-\sum_{i, j=1}^{n}\left(a_{i j}(x, t) z_{x_{i}}\right)_{x_{j}}=f_{y}(x, t, \bar{y}(x, t), \bar{u}(x, t)) z \\
\quad+f(x, t, \bar{y}(x, t), v(x, t))-f(x, t, \bar{y}(x, t), \bar{u}(x, t)), \quad \text { in } \Omega_{T} \\
\left.\quad z\right|_{\partial_{p} \Omega_{T}}=0 .
\end{array}\right.
$$

and

$$
\begin{aligned}
z^{0}= & \int_{\Omega_{T}} f_{y}^{0}(x, t, \bar{y}(x, t), \bar{u}(x, t)) z(x, t) d x d t \\
& +\int_{\Omega_{T}}\left[f^{0}(x, t, \bar{y}(x, t), v(x, t))-f^{0}(x, t, \bar{y}(x, t), \bar{u}(x, t))\right] d x d t
\end{aligned}
$$

We note that the solution $z$ of (4.23) and the quantity $z^{0}$ defined by (4.24) depend on the choice of $v \in \mathcal{U}$. Thus, we denote them by $z(\cdot, \cdot ; v)$ and $z^{0}(v)$. respectively. Then, taking limits in (4.16), we obtain

$$
\varphi^{0} z^{0}(v)+\left\langle\varphi, G^{\prime}(\bar{y}) z(\cdot, \cdot ; v)\right\rangle \geq 0, \quad \forall v \in \mathcal{U}
$$

Now, we let

$$
\psi^{0}=-\varphi^{0} \in[-1,0]
$$

Then, (2.11) follows from (4.21). Also, we obtain (2.13) by taking limits in (4.20) (along the above-mentioned subsequence). Furthermore, (4.25) can be written as

$$
\psi^{0} z^{0}(v)-\left\langle G^{\prime}(\bar{y})^{*} \varphi, z(\cdot, \cdot ; v)\right\rangle \leq 0, \quad \forall v \in \mathcal{U}
$$

We note that $G^{\prime}(\bar{y})^{*} \varphi \in \mathcal{M}\left(\bar{\Omega}_{T}\right)$ and by our compatible condition $(2.10)$, we see that

$$
\begin{gathered}
\left\langle G^{\prime}(\bar{y})^{*} \varphi, z\right\rangle=\left\langle\left.\left(G^{\prime}(\bar{y})^{*} \varphi\right)\right|_{\Omega_{T}}, z\right\rangle_{\mathcal{M}\left(\Omega_{T}\right), C\left(\bar{\Omega}_{T}\right)} \\
+\left\langle\left.\left(G^{\prime}(\bar{y})^{*} \varphi\right)\right|_{\Omega \times\{T\}},\left.z\right|_{t=T}\right\rangle_{\mathcal{M}(\Omega), C(\bar{\Omega})} .
\end{gathered}
$$

By [1], we know that (2.12) admits a solution in $L^{q}\left(0, T, W_{0}^{1, q}(\Omega)\right)$ for any $1<q<\frac{n+2}{n+1}$. However, unlike the elliptic equations, the function $z(x, t ; v)$ is not smooth enough (in $t$ direction) to be a test function for the equation (2.12). We shall get around this problem by approximating the equations for $z$ and the equations (2.12). 
We consider the following approximation for $z(x, t ; v)$ :

$$
\left\{\begin{array}{l}
z_{t}^{\delta}-\sum_{i, j=1}^{n}\left(a_{i j}^{\delta}(x, t) z_{x_{i}}^{\delta}\right)_{x_{j}}=f_{y}(x, t, \bar{y}(x, t), \bar{u}(x, t)) z^{\delta} \\
\quad+f(x, t, \bar{y}(x, t), v(x, t))-f(x, t, \bar{y}(x, t), \bar{u}(x, t)), \quad \text { in } \Omega_{T}, \\
\left.z^{\delta}\right|_{\partial_{p} \Omega_{T}}=0,
\end{array}\right.
$$

where $a_{i j}^{\delta} \in C^{2,1}\left(\bar{\Omega}_{T}\right), a_{i j}^{\delta}$ satisfies (A1), and

$$
a_{i j}^{\delta} \rightarrow a_{i j} \quad \text { in } L^{p}\left(\Omega_{T}\right), \text { as } \delta \rightarrow 0,
$$

for any $1<p<\infty$. By $L^{p}$ estimates for the parabolic equations, $z^{\delta}=z^{\delta}(\cdot, \cdot ; v) \in$ $W_{p}^{2,1}\left(\Omega_{T}\right)$ for any $1<p<\infty$. As before, we have the estimates

$$
\left\|z^{\delta}\right\|_{C^{\beta, \beta / 2}\left(\bar{\Omega}_{T}\right)}+\left\|\nabla_{x} z^{\delta}\right\|_{L^{2}\left(\Omega_{T}\right)} \leq C .
$$

where the constants $C$ and $\beta$ are independent of $\delta$ and $v \in \mathcal{U}$. Thus, by compactness and the uniqueness of the equation (4.23), one can easily derive that

$$
\left\|z^{\delta}-z\right\|_{C\left(\bar{\Omega}_{T}\right)} \rightarrow 0, \quad \text { as } \delta \rightarrow 0 .
$$

Clearly, (4.27) and (4.32) imply that

$$
\lim _{\delta \rightarrow 0}\left\{\psi^{0} z^{0}(v)-\left\langle G^{\prime}(\bar{y})^{*} \varphi, z^{\delta}(\cdot, \cdot ; v)\right\rangle\right\} \leq 0, \quad \forall v \in \mathcal{U}
$$

By [1], if we replace $a_{i j}$ with $a_{i j}^{\delta}$, then $(2.12)$ has a solution $\psi^{\delta} \in L^{q}\left(0, T, W_{0}^{1, q}(\Omega)\right)$, where $1<q<\frac{n+2}{n+1}$. ( $\psi^{\delta}$ is actually unique.) Furthermore,

$$
\left\|\psi^{\delta}\right\|_{L^{q}\left(0, T, W_{0}^{1, q}(\Omega)\right)} \leq C,
$$

where the constant $C$ is independent of $\delta$. Clearly, $v$ is not involved in the definition of $\psi^{\delta}$. By passing to a subsequence if necessary, we have, as $\delta \rightarrow 0$,

$$
\psi^{\delta} \stackrel{w}{\rightarrow} \psi \quad \text { in } L^{q}\left(\Omega_{T}\right), \quad \psi_{x_{j}}^{\delta} \stackrel{w}{\longrightarrow} \psi_{x_{j}} \quad \text { in } L^{q}\left(\Omega_{T}\right),
$$

for some function $\psi$. It follows that $\psi$ is a solution of (2.12). 
Since $z^{\delta} \in W_{p}^{2,1}\left(\Omega_{T}\right)$ for $p>q /(q-1)$, we can use $z^{\delta}$ as a test function in the equation for $\psi^{\delta}$.

Then, by some direct computation, we can reduce (4.33) to the following:

$$
\begin{aligned}
& \int_{\Omega_{T}}\left\{\psi^{0}\left[f^{0}(x, t, \bar{y}(x, t), \bar{u}(x, t))-f^{0}(x, t, \bar{y}(x, t), v(x, t))\right]\right. \\
& \left.\quad+\psi^{\delta}(x, t)[f(x, t, \bar{y}(x, t), \bar{u}(x, t))-f(x, t, \bar{y}(x, t), v(x, t))]\right\} d x \\
& \geq o(1), \quad \text { as } \delta \rightarrow 0 .
\end{aligned}
$$

Now letting $\delta \rightarrow 0$ and recalling (4.35), we obtain,

$$
\begin{aligned}
& \int_{\Omega_{T}}\left\{\psi^{0}\left[f^{0}(x, t, \bar{y}(x, t), \bar{u}(x, t))-f^{0}(x, t, \bar{y}(x, t), v(x, t))\right]\right. \\
& +\psi(x, t)[f(x, t, \bar{y}(x, t), \bar{u}(x, t))-f(x, t, \bar{y}(x, t), v(x, t))]\} d x \\
& \equiv \int_{\Omega}\left[H\left(x, t, \bar{y}(x, t), \bar{u}(x, t), \psi^{0}, \psi(x, t)\right)-H\left(x, t, \bar{y}(x, t), v(x, t), \psi^{0}, \psi(x, t)\right)\right] d x, \\
& \geq 0, \quad \forall v \in \mathcal{U} .
\end{aligned}
$$

Then, by the separability of $U$ and the continuity of the Hamiltonian $H$ in the variable $v$, noticing also that $v \in \mathcal{U}$ is arbitrary, we obtain the maximum condition (2.14) (see $[5])$.

\section{$\S 5$. Applications.}

In this section, we would like to discuss some special cases which are covered by our main result.

We first consider the following case. Let $Z=C_{0}\left(\bar{\Omega}_{T}\right)$ with some norm $|\cdot|_{0}$ which is equivalent to $\|\cdot\|_{C\left(\bar{\Omega}_{T}\right)}$ and the dual of it, still denoted by $\mathcal{M}_{0}\left(\bar{\Omega}_{T}\right)$, is strictly convex. We let $Q \subset Z$ be defined as in (1.3) and $g: \bar{\Omega}_{T} \times \mathbb{R} \rightarrow \mathbb{R}$ be continuous with $g_{y}(x, t, y)$ being also continuous. Moreover,

$$
\left\{\begin{array}{l}
g(x, t, 0)<0, \quad \forall(x, t) \in \partial \Omega \times[0, T], \\
g\left(x, 0, y_{0}(x)\right)<0, \quad \forall x \in \bar{\Omega},
\end{array}\right.
$$

where $y_{0} \in C_{0}(\bar{\Omega})$. We let $G(\eta)=g(x, t, \eta(x, t))$, for any $\eta \in C_{0}\left(\bar{\Omega}_{T}\right)$. Then, the following result holds.

Proposition 5.1. For the above $Q, G$ and $y_{0}$, condition (2.10) holds. 
Proof. By (5.1), we see that for any $\varepsilon>0$, there exists a $\delta>0$, such that

$$
\begin{aligned}
g(x, t, y) \leq-\varepsilon, & \text { if } t \in[\delta, T],|y|<\delta, d(x, \partial \Omega)<\delta, \\
& \text { or } t \in[0, \delta], x \in \Omega,\left|y-y_{0}(x)\right|<\delta .
\end{aligned}
$$

Thus, for any $\eta \in C_{0}\left(\bar{\Omega}_{T}\right)$ with $G(\eta) \in Q$ and $\left.\eta\right|_{t=0}=y_{0}(x)$, we have the following: For any $\varepsilon>0$, there exists a $\delta>0$, such that

$$
g(x, t, \eta(x, t)) \leq-\varepsilon, \quad \text { if } d\left((x, t), \partial_{p} \Omega_{T}\right)<\delta
$$

Now, for any $\varphi \in C\left(\bar{\Omega}_{T}\right)$, if for some $\delta>0$, it holds

$$
\operatorname{supp} \varphi \subset\left\{(x, t) \in \bar{\Omega}_{T} \mid d\left((x, t), \partial_{p} \Omega_{T}\right) \leq \delta\right\}
$$

then, for all small enough $\sigma>0$, we have

$$
g(x, t, \eta(x, t))+\sigma \varphi(x, t) \leq 0, \quad(x, t) \in \bar{\Omega}_{T} .
$$

This means $G(\eta)+\sigma \varphi \in Q$ for all small $\sigma>0$. Hence, by the definition of the generalized gradient, we obtain

$$
\langle\zeta, \varphi\rangle=0, \quad \forall \zeta \in \partial d_{Q}(G(\eta)) \subset \mathcal{M}\left(\bar{\Omega}_{T}\right)
$$

In another word, we have (for the above $\eta$ )

$$
\operatorname{supp} \zeta \subset\left\{(x, t) \in \bar{\Omega}_{T} \mid d\left((x, t), \partial_{p} \Omega_{T}\right) \geq \delta\right\}
$$

Since $G^{\prime}(\eta)=g_{y}(x, t, \eta(x, t)) I$, with $I$ being the identity on $Z$, we see that $(2.10)$ holds.

We have already know that $Q$ has a nonempty interior in $Z$, hence it is of codimension 0 in $Z$. Then, our main result is applicable to this case. Let us state the corresponding result.

Theorem 5.2. Let $(\bar{y}, \bar{u})$ be an optimal pair. Then, there exists a constant $\psi^{0} \leq 0$, a function $\psi \in L^{q}\left(0, T ; W_{0}^{1, q}(\Omega)\right)\left(q<\frac{n+2}{n+1}\right)$ and a $\varphi \in \mathcal{M}_{0}\left(\bar{\Omega}_{T}\right)$, such that

$$
\left|\psi^{0}\right|+\|\varphi\|_{\mathcal{M}_{0}\left(\bar{\Omega}_{T}\right)}>0
$$




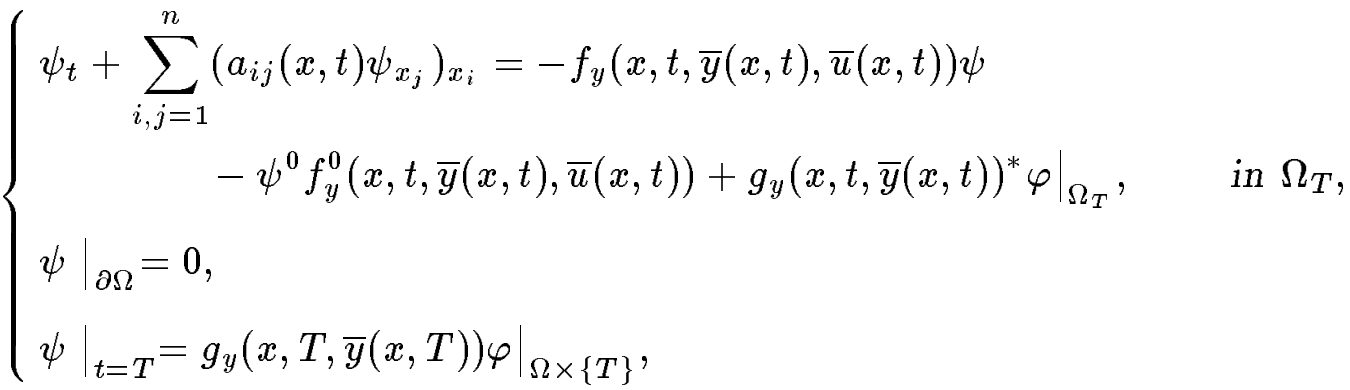

$$
\begin{aligned}
& \int_{\bar{\Omega}_{T}}[z(x, t)-g(x, t, \bar{y}(x, t))] d \varphi(x, t) \leq 0, \quad \forall z \in Q \\
& H\left(x, t, \bar{y}(x, t), \bar{u}(x, t), \psi^{0}, \psi(x, t)\right)=\max _{v \in U} H\left(x, t, \bar{y}(x, t), v, \psi^{0}, \psi(x, t)\right), \\
& \text { a.e. }(x, t) \in \Omega \times[0, T] \text {. }
\end{aligned}
$$

where $H$ is the Hamiltonian defined by (2.15).

Let us make some further remark on the above result. We set

$$
\Omega_{T}^{0}=\left\{(x, t) \in \bar{\Omega}_{T} \mid g(x, t, \bar{y}(x, t))=0\right\} .
$$

Then, by our condition (5.1), we see that

$$
\Omega_{T}^{0} \subset \Omega_{T} \bigcup(\Omega \times\{T\}) .
$$

The set $\Omega_{T}^{0}$ is called the active set for the optimal state $\bar{y}$. We have that

$$
\operatorname{supp} \varphi \subset \Omega_{T}^{0}
$$

In fact, for any $\eta \in C\left(\bar{\Omega}_{T}\right)$ with supp $\eta \subset \bar{\Omega}_{T} \backslash \Omega_{T}^{0}, g(\cdot, \cdot, \bar{y}(\cdot, \cdot)) \pm \varepsilon \eta \in Q$ if $\varepsilon$ is small enough. By the transversality condition (5.10), we see immediately that

$$
\int_{\bar{\Omega}_{T}} \eta(x, t) d \varphi(x, t)=0 .
$$

This gives (5.14).

The above situation is comparable with the case discussed in [5] for quasilinear elliptic equations. 
Next, let us look at another important case. Let $Z=C_{0}\left(\bar{\Omega}_{T}\right)$ as before and let $\left(x_{i}, t_{i}\right) \in \Omega_{T} \bigcup(\Omega \times\{T\})(1 \leq i \leq m)$ be given $m$ (different) points and also let $b_{i} \in \mathbb{R}, 1 \leq$ $i \leq m$. We define $Q$ as in (1.5). Then, we see that $Q$ is a finite codimensional convex and closed subset of $Z$. Also, it is not hard to see that for any $\eta \in Q$ and any $\zeta \in \partial d_{Q}(\eta)$, we have

$$
\zeta=\sum_{i=1}^{m} \lambda_{i} \delta_{\left(x_{i} t_{i}\right)}
$$

where $\lambda_{i} \in \mathbb{R}$ and $\delta_{\left(x_{i}, t_{i}\right)}$ is the Dirac measure concentrated at point $\left(x_{i}, t_{i}\right)$ with mass 1. Thus, we see that condition (2.10) holds (in the present case $G=I$ the identity). Hence, our result applies to this situation. Let us state the corresponding result below.

Theorem 5.3. Let $(\bar{y}, \bar{u})$ be an optimal pair. Then, there exists a constant $\psi^{0} \leq 0$, a function $\psi \in L^{q}\left(0, T ; W_{0}^{1, q}(\Omega)\right)\left(q<\frac{n+2}{n+1}\right)$ and real numbers $\lambda_{i}, 1 \leq i \leq m$, such that

$$
\begin{aligned}
& \left\{\begin{array}{l}
\psi_{t}+\sum_{i, j=1}^{n}\left(a_{i j}(x, t) \psi_{x_{j}}\right)_{x_{i}}=-f_{y}(x, t, \bar{y}(x, t), \bar{u}(x, t)) \psi \\
\quad-\psi^{0} f_{y}^{0}(x, t, \bar{y}(x, t), \bar{u}(x, t))+\sum_{t_{i}<T} \lambda_{i} \delta_{\left(x_{i}, t_{i}\right)}, \quad \text { in } \Omega_{T},
\end{array}\right. \\
& \psi \begin{array}{l}
\left.\psi\right|_{\partial \Omega}=0, \\
\left.\psi\right|_{t=T}=\sum_{t_{i}=T} \lambda_{i} \delta_{\left(x_{i}, t_{i}\right)},
\end{array} \\
& H\left(x, t, \bar{y}(x, t), \bar{u}(x, t), \psi^{0}, \psi(x, t)\right)=\max _{v \in U} H\left(x, t, \bar{y}(x, t), v, \psi^{0}, \psi(x, t)\right), \\
& \text { a.e. }(x, t) \in \Omega \times[0, T] .
\end{aligned}
$$

Next, let us point out some other state constraints, which are covered by our general result.

$1^{\circ}$. Let $Z=L^{p}\left(\Omega_{T}\right), 1<p<\infty . F: \Omega_{T} \times \mathbb{R} \rightarrow \mathbb{R}$ and

$$
\left\{\begin{array}{l}
Q=\left\{z \in L^{p}\left(\Omega_{T}\right) \mid \int_{\Omega_{T}} z(x, t) d x d t \leq 0\right\}, \\
G(\eta)(x, t)=F(x, t, \eta(x, t)), \quad \forall \eta \in C_{0}\left(\bar{\Omega}_{T}\right) .
\end{array}\right.
$$


Then, the corresponding state constraint is

$$
\int_{\Omega_{T}} F(x, t, \eta(x, t)) d x d t \leq 0
$$

$2^{\circ}$. Let $Z=L^{p}\left(\Omega_{T}\right)^{m}, 1<p<\infty, F_{i}: \Omega_{T} \times \mathbb{R} \rightarrow \mathbb{R}, 1 \leq i \leq m$,

$$
\left\{\begin{array}{l}
Q=\left\{z \in L^{p}\left(\Omega_{T}\right)^{m} \mid \int_{\Omega_{T}} z(x, t) d x d t=0\right\} \\
G(\eta)(x, t)=\left(F_{1}(x, t, \eta(x, t)), \cdots, F_{m}(x, t, \eta(x, t)), \quad \forall \eta \in C_{0}\left(\bar{\Omega}_{T}\right) .\right.
\end{array}\right.
$$

Then, the state constraint is

$$
\int_{\Omega_{T}} F_{i}(x, t, y(x, t)) d x d t=0, \quad 1 \leq i \leq m
$$

$3^{\circ}$. Let $Z=C_{0}\left(\bar{\Omega}_{T}\right), F: \bar{\Omega}_{T} \times \mathbb{R} \rightarrow \mathbb{R}$ such that

$$
F\left(x_{i}, t_{i}, y\right)=g_{i}(y), \quad 1 \leq i \leq m
$$

where $\left(x_{i}, t_{i}\right) \in \Omega_{T} \bigcup(\Omega \times\{T\})$ are given different points. Let

$$
\left\{\begin{array}{l}
Q=\left\{z \in C_{0}\left(\bar{\Omega}_{T}\right) \mid z\left(x_{i}, t_{i}\right)=b_{i}, \quad 1 \leq i \leq m\right\} \\
G(\eta)(x, t)=F(x, t, \eta(x, t)), \quad \forall \eta \in C_{0}\left(\bar{\Omega}_{T}\right)
\end{array}\right.
$$

Then, the state constraint is

$$
g_{i}\left(y\left(x_{i}, t_{i}\right)\right)=b_{i}, \quad 1 \leq i \leq m
$$

This is a generalization of (1.6).

$4^{\circ}$. Let $Z=C_{0}\left(\bar{\Omega}_{T}\right), F: \bar{\Omega}_{T} \times \mathbb{R} \rightarrow \mathbb{R}$ such that (5.24) holds. Let

$$
\left\{\begin{array}{l}
Q=\left\{z \in C_{0}\left(\bar{\Omega}_{T}\right) \mid z\left(x_{i}, t_{i}\right)=z\left(x_{j}, t_{i}\right), \quad 1 \leq i, j \leq m\right\} \\
G(\eta)(x, t)=F(x, t, \eta(x, t)), \quad \forall \eta \in C_{0}\left(\bar{\Omega}_{T}\right)
\end{array}\right.
$$

Then, the state constraint is

$$
g_{i}\left(y\left(x_{i}, t_{i}\right)\right)=g_{j}\left(y\left(x_{j}, t_{j}\right)\right), \quad 1 \leq i, j \leq m
$$


In particular, if we take $F(x, t, y)=y$, then, (5.29) means

$$
y\left(x_{i}, t_{i}\right)=y\left(x_{j}, t_{j}\right), \quad 1 \leq i, j \leq m
$$

Physically, this means that we want the temperatures, say, at points $\left(x_{i}, t_{i}\right)$ are the same.

There are many other examples. But, we prefer to omit them here. We should point out that the pointwise constraint like (1.6) is actually an approximation of the constraint like

$$
\left.\mid y\left(x_{i}, t\right) i\right)-b_{i} \mid \leq \varepsilon, \quad 1 \leq i \leq m
$$

with $\varepsilon>0$ being very small. Physically, this means, for example, that the temperature at point $\left(x_{i}, t_{i}\right)$ has to be controlled near $b_{i}$ with an accuracy $\varepsilon$.

\section{Quasilinear Parabolic Equations.}

Finally, let us remark that the result extends to the quasilinear parabolic equations as those considered in [5] for elliptic cases. The Hölder estimates for the gradient (in $x$ direction) of the solution is required, which is available [16], when the leading coefficients is assumed to be Hölder continuous in $x$ direction. After a careful examination of the proof of Lemma 3.2, we conclude that $a_{i j}$ can actually be allowed to depend on $\rho$.

We consider the equations

$$
\left\{\begin{array}{l}
\zeta_{t}-\left(a_{i j}^{\rho}(x, t) \zeta_{x_{i}}\right)_{x_{j}}+c_{\rho}(x, t) \zeta(x, t)=\left(1-\frac{1}{\rho} \chi_{E_{\rho}}(x, t)\right) h(x, t), \\
\zeta=0, \quad(x, t) \in \partial_{p} \Omega_{T} .
\end{array}\right.
$$

It is clear that the solution $\zeta=\zeta_{E_{\rho}}(x, t)$ is uniquely determined by the choice of the coefficients $a_{i j}^{\rho}, c_{\rho}$ and the set $E_{\rho}$. Let $K>0,0<\lambda<\Lambda$ and

$$
\begin{aligned}
\mathcal{K}=\left\{\left(a_{i j}(x, t), c(x, t)\right) ;\right. & \|c\|_{L^{\infty}\left(\Omega_{T}\right)} \leq K \\
& \left.\lambda|\xi|^{2} \leq a_{i j}(x, t) \xi_{i} \xi_{j} \leq \Lambda|\xi|^{2}, \forall(x, t) \in \bar{\Omega}_{T}, \xi \in \mathbb{R}^{n}\right\}
\end{aligned}
$$

Lemma 6.1. Suppose that $\frac{n+2}{2}<p<\infty$. Then, there exists $\theta \in(0,1)$ such that for each $h^{0} \in L^{1}\left(\Omega_{T}\right), h \in L^{p}\left(\Omega_{T}\right), K>0$, and any $\rho \in(0,1)$

$$
\inf _{E_{\rho} \in \mathcal{E}_{\rho}\left(a_{i j}^{\rho}, c_{\rho}\right) \in \mathcal{K}}\left\{\left|\int_{0}^{T} \int_{\Omega}\left(1-\frac{1}{\rho} \chi_{E_{\rho}}(x, t)\right) h^{0}(x, t) d x d t\right|+\left\|\zeta_{E_{\rho}}\right\|_{C^{\theta, \theta / 2}(\bar{\Omega} \times[0, T])}\right\}=0 .
$$


We now consider the following parabolic equation:

$$
\left\{\begin{array}{l}
y_{t}-\nabla_{x}\left(a\left(x, t, \nabla_{x} y\right)\right)=f(x, t, y, u(t, x)), \quad \text { in } \Omega_{T}, \\
\left.y\right|_{\partial \Omega}=0, \\
\left.y\right|_{t=0}=y_{0}(x), \quad x \in \Omega,
\end{array}\right.
$$

where $a$ satisfies

(A1) The functions $a: \bar{\Omega}_{T} \times \mathbb{R}^{n} \rightarrow \mathbb{R}^{n}$ and $\frac{\partial a_{i}}{\partial p_{j}}: \bar{\Omega}_{T} \times \mathbb{R}^{n} \rightarrow \mathbb{R}$ are continuous. There exist $\Lambda>\lambda>0, \Lambda_{1}>0, \alpha \in(0,1)$ and $m>1$ such that

$$
\left\{\begin{array}{l}
\frac{\partial a_{i}}{\partial p_{j}}(x, t, p) \xi_{i} \xi_{j} \geq \lambda(1+|p|)^{m-2}|\xi|^{2}, \quad \forall(x, t, p) \in \bar{\Omega} \times[0, T] \times \mathbb{R}^{n}, \\
\left|\frac{\partial a_{i}}{\partial p_{j}}(x, t, p)\right| \leq \Lambda(1+|p|)^{m-2}, \quad \forall(x, t, p) \in \bar{\Omega} \times[0, T] \times \mathbb{R}^{n},
\end{array}\right.
$$

and

$$
\begin{aligned}
&|a(x, t, p)-a(\widetilde{x}, t, p)| \leq \Lambda_{1}(1+|p|)^{m-1}|x-\widetilde{x}|^{\alpha}, \\
& \forall(x, t),(\widetilde{x}, t) \in \bar{\Omega} \times[0, T], p \in \mathbb{R}^{n} .
\end{aligned}
$$

Under the assumptions $(\overline{\mathrm{A} 1})$ and (A2), (6.4) has a unique solution $y(x, t)$ such that $y, y_{x_{j}} \in$ $C^{\beta, \beta / m}\left(\bar{\Omega}_{T}\right)$, for any $y_{0} \in C^{1+\alpha}(\bar{\Omega}) \cap C_{0}(\bar{\Omega})$ (see [16]). The a-priori estimates for $y$ and $y_{x_{j}}$ in the space $C^{\beta, \beta / m}\left(\bar{\Omega}_{T}\right)$ are valid uniformly for $u \in \mathcal{U}$. If $u, \widehat{u} \in \mathcal{U}$ and $y, \widehat{y}$ are the corresponding states, then by using an interpolation theorem of the $C^{k+\alpha}$ spaces, we have, for any $0<\theta<\beta$,

$$
\|y-\widehat{y}\|_{C\left(\bar{\Omega}_{T}\right)}+\left\|\nabla_{x}(y-\widehat{y})\right\|_{C^{\theta, \theta / m}\left(\bar{\Omega}_{T}\right)} \rightarrow 0, \quad \text { uniformly as } \bar{d}(u, \widehat{u}) \rightarrow 0 .
$$

Lemma 6.1 and (6.7) are the essential estimates needed to establish the variation theorem similar to Theorem 3.3. We have

Lemma 6.2. The same statement of Theorem 3.3 is still valid for the quasilinear case, except that the $a_{i j}(x, t)$ in (3.25) should be replaced by $\bar{a}_{i j}(x, t) \equiv \frac{\partial a_{i}}{\partial p_{j}}\left(x, t, \nabla_{x} y(x, t)\right)$.

Sketch of Proof. The proof of Theorem 3.3 also goes through, where we shall replace $a_{i j}(x, t)$ in (3.32) with $a_{i j}^{\rho}(x, t) \equiv \int_{0}^{1} \frac{\partial a_{i}}{\partial p_{j}}\left(x, t, \nabla_{x} y(x, t)+\tau \nabla_{x}\left(y_{\rho}(x, t)-y(x, t)\right) d \tau\right.$. Using (6.7), we can easily obtain

$$
a_{i j}^{\rho} \rightarrow \bar{a}_{i j}, \quad \text { in } C\left(\bar{\Omega}_{T}\right)
$$


Write $z_{\rho}-z \equiv\left(z_{\rho}-\widehat{z}_{\rho}\right)+\left(\widehat{z}_{\rho}-z\right)$, where $\widehat{z}_{\rho}$ satisfies the equation

$$
\left\{\begin{array}{l}
\left(\widehat{z}_{\rho}\right)_{t}-\sum_{i, j=1}^{n}\left(a_{i j}^{\rho}(x, t)\left(\widehat{z}_{\rho}\right)_{x_{i}}\right)_{x_{j}}+c(x, t) \widehat{z}_{\rho}=h(x, t), \quad \text { in } \Omega_{T}, \\
\left.\widehat{z}_{\rho}\right|_{\partial_{p} \Omega_{T}}=0 .
\end{array}\right.
$$

We can treat $z_{\rho}-\widehat{z}_{\rho}$ the same way as before, apply Lemma 6.1 instead of Lemma 3.2, and then obtain

$$
\left\|z_{\rho}-\widehat{z}_{\rho}\right\|_{C^{\theta, \theta / 2}\left(\bar{\Omega}_{T}\right)} \rightarrow 0 \quad \text { as } \rho \rightarrow 0
$$

for a special choice of $E_{\rho}$ with $\left|E_{\rho}\right|=\rho\left|\Omega_{T}\right|$. Clearly, by Hölder's estimates [12, Chapter III, section 10$]$, for $\widehat{\zeta}_{\rho}=\widehat{z}_{\rho}-z$,

$$
\left\|\widehat{\zeta}_{\rho}\right\|_{C^{\beta, \beta / 2}\left(\bar{\Omega}_{T}\right)} \leq\left\|\widehat{z}_{\rho}\right\|_{C^{\beta, \beta / 2}\left(\bar{\Omega}_{T}\right)}+\|z\|_{C^{\beta, \beta / 2}\left(\bar{\Omega}_{T}\right)} \leq C
$$

and $\widehat{\zeta}_{\rho}$ satisfies the equation

$$
\left\{\begin{array}{l}
\left(\widehat{\zeta}_{\rho}\right)_{t}-\sum_{i, j=1}^{n}\left(a_{i j}^{\rho}(x, t)\left(\widehat{\zeta}_{\rho}\right)_{x_{i}}\right)_{x_{j}}+c(x, t) \widehat{\zeta}_{\rho}=\left(\left[a_{i j}^{\rho}(x, t)-\bar{a}_{i j}(x, t)\right] z_{x_{i}}(x, t)\right)_{x_{j}} \\
\left.\widehat{\zeta}_{\rho}\right|_{\partial_{p} \Omega_{T}}=0 .
\end{array}\right.
$$

Multiplying the above equation with $\widehat{\zeta}_{\rho}$ and integrating over $\Omega_{T}$ give us the usual energy estimates:

$$
\left\|\widehat{\zeta}_{\rho}\right\|_{L^{2}\left(\Omega_{T}\right)} \leq\left\|\nabla_{x} \widehat{\zeta}_{\rho}\right\|_{L^{2}\left(\Omega_{T}\right)} \leq C\left\|a_{i j}^{\rho}-\bar{a}_{i j}\right\|_{C\left(\bar{\Omega}_{T}\right)}\left\|\nabla_{x} z\right\|_{L^{2}\left(\Omega_{T}\right)} \rightarrow 0, \quad \text { as } \rho \rightarrow 0
$$

where we have used that fact that $\left\|\nabla_{x} z\right\|_{L^{2}\left(\Omega_{T}\right)} \leq C$, which is an easy consequence of the energy estimates for the solution $z(x, t)$.

Using the interpolation (Lemma 3.4), the estimates (6.11) and (6.13), we immediately obtain, for any $0<\theta<\beta$,

$$
\left\|\widehat{z}_{\rho}-z\right\|_{C^{\theta, \theta / 2}\left(\bar{\Omega}_{T}\right)}=\left\|\widehat{\zeta}_{\rho}\right\|_{C^{\theta, \theta / 2}\left(\bar{\Omega}_{T}\right)} \rightarrow 0, \quad \text { as } \rho \rightarrow 0
$$

Combining (6.10) and (6.14), we get an variation theorem like Theorem 3.3. 
Therefore, with the same argument as in section 4 (where we shall use (6.7) for the convergence of the leading coefficients), we have the following maximum principle:

Theorem 6.3. Consider the same problem except that the governing equation (1.1) is replaced by (6.4). Let $(\overline{A 1}),(A 2)$ and $(A 3)$ be in force. Suppose that $y_{0} \in C^{1+\alpha}(\bar{\Omega}) \cap C_{0}(\bar{\Omega})$ and that (2.10) holds. Let $(\bar{y}, \bar{u})$ be an optimal pair of Problem $C$ corresponding to the state equation (6.4). Then, there exists a constant $\psi^{0} \leq 0$, a function $\psi \in L^{q}\left(0, T ; W_{0}^{1, q}(\Omega)\right)$ $\left(1<q<\frac{n+2}{n+1}\right)$, and a $\varphi \in \partial d_{Q}(G(\bar{y})) \subset Z^{*}$, such that

$$
\left\{\begin{array}{l}
\psi_{t}+\sum_{i, j=1}^{n}\left(\frac{\partial a_{i}}{\partial p_{j}}\left(x, t, \nabla_{x} \bar{y}(x, t)\right) \psi_{x_{j}}\right)_{x_{i}}=-f_{y}(x, t, \bar{y}(x, t), \bar{u}(x, t)) \psi \\
\quad-\psi^{0} f_{y}^{0}(x, t, \bar{y}(x, t), \bar{u}(x, t))+\left.\left(G^{\prime}(\bar{y})^{*} \varphi\right)\right|_{\Omega_{T}}, \quad \text { in } \Omega_{T} \\
\left.\psi\right|_{\partial \Omega}=0 \\
\left.\psi\right|_{t=T}=\left.\left(G^{\prime}(\bar{y})^{*} \varphi\right)\right|_{\Omega \times\{T\}},
\end{array}\right.
$$

$$
\begin{aligned}
H\left(x, t, y, u, \psi^{0}, \psi\right)= & \psi^{0} f^{0}(x, t, y, u)+\psi f(x, t, y, u), \\
& \forall\left(x, t, y, u, \psi^{0}, \psi\right) \in \Omega \times[0, T] \times \mathbb{R} \times U \times \mathbb{R} \times \mathbb{R} .
\end{aligned}
$$

Remark 6.4. The semilinear case is not a special case of the quasilinear case, since the Hölder continuity of $a_{i j}$ (in $x$ direction) is not assumed in the semilinear case.

Acknowledgment. The authors thank Dr. Hong-Ming Yin for a stimulating discussion on this problem. 


\section{References}

[1] L. Boccardo and T. Gallouet, Non-linear elliptic and parabolic equations involving measure data, J. Functional Analysis, 87 (1989), 149-169.

[2] J. F. Bonnans and E. Casas, Un principe de Pontryagine pour le contrôle des systèmes semilinéaires elliptiques, J. Diff. Equ., 90 (1991), 288-303.

[3] J. F. Bonnans and E. Casas, A boundary Pontryagin's principle for the optimal control of state-constrained elliptic systems, Int. Ser. Numer. Math., 107 (1992), 241-249.

[4] J. F. Bonnans and E. Casas, An extension of Pontryagin's principle for state-constrained optimal control of semilinear elliptic equations and variational inequalities, submitted.

[5] E. Casas and J. Yong, Maximum principle for state-constrained optimal control problems governed by quasilinear elliptic equations, IMA Preprint, \# 1063.

[6] F. H. Clarke, Optimization and Nonsmooth Analysis, SIAM, 1990.

[7] J. Diestel, Geometry of Banach Spaces - Selected Topics, Lecture Notes in Math. No.485, Springer-Verlag, Berlin, 1975.

[8] I. Ekeland, Nonconvex minimization problems, Bull. Amer. Math. Soc. (New Series), 1(1979), 443-474.

[9] H.O. Fattorini, A unified theory of necessary conditions for nonlinear nonconvex control systems, Appl. Math. Optim., 15 (1987), 141-185.

[10] H.O. Fattorini and H. Frankowska, Necessary conditions for infinite dimensional control problems, Math. Control Signal Systems, 4 (1991), 41-67.

[11] D. Gilbarg and N. S. Trudinger, Elliptic Partial Differential Equations of Second Order, 2nd Edition, Springer-Verlag, 1983.

[12] O.A. Ladyženskaja, V.A. Solonnikov and N.N. Uralćeva Linear and quasi-linear equations of parabolic type, Transl. Math. Monographs., Vol 23, 1968. 
[13] X. Li, Vector-valued measure and the necessary conditions for the optimal control problems of linear systems, Proc. IFAC 3rd Symposium on Control of Distributed Parameter Systems, Toulouse, France, 1982,

[14] X. Li and Y. Yao, Maximum principle of distributed parameter systems with time lags, Lecture Notes in Control \& Inform. Sci., Springer-Verlag, Berlin, Vol. 75 (1985), 410427.

[15] X. Li and J. Yong, Necessary conditions of optimal control for distributed parameter systems, SIAM J. Control \& Optim., 29 (1991), 895-908.

[16] G. M. Lieberman, Boundary and initial regularity for solutions of degenerate parabolic Equations, Nonlinear Anal., TMA, Vol. 29 (1993), 551-569.

[17] J. L. Lions, Optimal Control of Systems Governed by Partial Differential Equations, Springer-Verlag, New York, 1971

[18] L. Pan and J. Yong, Optimal control for quasilinear retarded parabolic systems, Math. Systems, Estimations \& Control, to appear.

[19] L. S. Pontryagin, V. G. Boltyanskii, R. V. Gamkrelidze and E. F. Mischenko, Mathematical Theory of Optimal Processes, Wiley, New York, 1962.

[20] J. Yong, Pontryagin maximum principle for semilinear second order elliptic partial differential equations and variational inequalities with state constraints, Diff. Int. Equ., 5 (1992), 1307-1334. 